\title{
On the Quantum Homology of Real Lagrangians in Fano Toric Manifolds
}

\section{Luis Haug}

Department of Mathematics, ETH Zürich, Rämistrasse 101, 8092 Zürich, Switzerland

Correspondence to be sent to: haug@math.ethz.ch

Let $R$ be the real Lagrangian in a toric symplectic manifold $X$, the fixed point set under complex conjugation. Assuming that $X$ is Fano of minimal Chern number at least 2, we show that the Lagrangian quantum homology $\mathrm{OH}(R ; \Lambda)$ of $R$ over the ring $\Lambda=\mathbb{Z}_{2}\left[t, t^{-1}\right]$ is isomorphic to $H\left(R ; \mathbb{Z}_{2}\right) \otimes \Lambda$ as a module. Moreover, we prove that $\mathrm{OH}(R ; \Lambda)$ is isomorphic as a ring to the quantum homology $\mathrm{OH}(X ; \Lambda)$ of the ambient symplectic manifold.

\section{Introduction}

\subsection{Setting}

Let $(X, \omega)$ be a symplectic toric manifold of dimension $2 n$, that is, a compact symplectic manifold endowed with an effective Hamiltonian action of an $n$-dimensional torus $Q$. Denote by $\mu: X \rightarrow \mathfrak{q}^{*}$ its moment map, where $\mathfrak{q}^{*}$ is the dual of the Lie algebra of $Q$, and by $\Delta$ its moment polytope, that is, the image of $\mu$. $X$ carries a distinguished anti-symplectic involution $\tau: X \rightarrow X$, characterized by the fact that it leaves the moment map $\mu$ invariant. We refer to it as complex conjugation. Its fixed point set

$$
R:=\operatorname{Fix} \tau
$$

is a Lagrangian submanifold, which we call the real Lagrangian of $X$. The prototypical example for a pair $(X, R)$ is $\left(\mathbb{C} P^{n}, \mathbb{R} P^{n}\right)$. 
For the purpose of this paper, we think of a symplectic toric manifold as being obtained by equipping a Fano toric manifold $X$ with a Kähler form $\omega$ whose cohomology class represents a positive multiple of $c_{1}(X)$. In particular, we thus assume $\omega$ to be monotone, which by definition means that the homomorphisms $I_{\omega}, I_{C_{1}}: \pi_{2}(X) \rightarrow \mathbb{R}$ given by integration of the classes $\omega$ and $C_{1}(X)$ satisfy

$$
I_{C_{1}}=\rho I_{\omega}
$$

for some $\rho \geq 0$. This ensures that $R$ is also monotone, meaning in this case that the homomorphisms $I_{\omega}, I_{\mu}: \pi_{2}(X, R) \rightarrow \mathbb{R}$ given by integration of $\omega$, respectively, the Maslov index are related by

$$
I_{\omega}=\rho^{\prime} I_{\mu}
$$

for some $\rho^{\prime} \geq 0$. The minimal Chern number of $X$ and the minimal Maslov number of $R$ are defined by

$$
\begin{aligned}
& C_{X}:=\min \left\{\left\langle C_{1}(X), \lambda\right\rangle>0 \mid \lambda \in \pi_{2}(X)\right\}, \\
& N_{R}:=\min \left\{\langle\mu(R), \lambda\rangle>0 \mid \lambda \in \pi_{2}(X, R)\right\} .
\end{aligned}
$$

It is easy to see that they coincide in our situation, $C_{X}=N_{R}$. We assume further that

$$
C_{X}, N_{R} \geq 2
$$

\subsection{Main result}

Under these assumptions, various versions of the Lagrangian quantum homology ring $\mathrm{QH}(R)$ introduced by Biran and Cornea [7] are defined. The purpose of this paper is to study

$$
\mathrm{OH}\left(R ; \Lambda_{R}\right),
$$

the version obtained by using as coefficients the ring $\Lambda_{R}=\mathbb{Z}_{2}\left[t^{-1}, t\right]$ of $\mathbb{Z}_{2}$-Laurent polynomials, the latter graded by setting the degree of the formal variable to be $|t|=-N_{R}$. We will show that there is a close relation to the quantum homology ring 
of the ambient manifold defined over the ring $\Lambda_{X}=\mathbb{Z}_{2}\left[q^{-1}, q\right]$, where in this case the formal variable has degree $|q|=-2 C_{X}$.

The main result of the paper is the following theorem.

Theorem A. Let $(X, \omega)$ be a monotone symplectic toric manifold with minimal Chern number $C_{X} \geq 2$ and let $R$ be its real Lagrangian. Then the following hold.

(i) There exists a canonical isomorphism of $\Lambda_{R}$-modules

$$
\mathrm{QH}\left(R ; \Lambda_{R}\right) \cong H\left(R ; \mathbb{Z}_{2}\right) \otimes \Lambda_{R}
$$

(ii) Moreover, there exists a canonical degree-doubling ring isomorphism

$$
\mathrm{OH}\left(R ; \Lambda_{R}\right) \cong \mathrm{OH}\left(X ; \Lambda_{X}\right)
$$

The first statement says that $R$ is $\Lambda_{R}$-wide in the terminology of Biran and Cornea [7], meaning the existence of an isomorphism as in (i) (in general not required to be canonical). Since $\mathrm{OH}\left(R ; \Lambda_{R}\right)$ is isomorphic to the Lagrangian intersection Floer homology $\mathrm{HF}\left(R ; \Lambda_{R}\right)$, see [7], the wideness immediately implies the following nondisplacement result: for any Hamiltonian diffeomorphism $\phi$ of $(X, \omega)$ such that $R$ and $\phi(R)$ intersect transversely, we have

$$
\#(R \cap \phi(R)) \geq \sum_{j=1}^{n} b_{j}\left(R ; \mathbb{Z}_{2}\right),
$$

where the $b_{j}\left(R ; \mathbb{Z}_{2}\right)$ denote the $\mathbb{Z}_{2}$ Betti numbers of $R$. In terms of the moment polytope $\Delta$, the right-hand side is the number of its vertices, as will become clear later.

The second part of the theorem allows to write down the ring structure of $\mathrm{OH}\left(R ; \Lambda_{R}\right)$ using the known description of $\mathrm{OH}\left(X ; \Lambda_{X}\right)$ for Fano toric manifolds. The latter was first computed by Batyrev [5] and later by Cieliebak and Salamon [11] using different methods (both work with more general coefficients). We give two examples.

Example 1.1. Consider $X=\mathbb{C} P^{n}$ with its standard monotone symplectic form and its real part $R=\mathbb{R} P^{n}$. We have $C_{X}=N_{R}=n+1$. Denote by $h_{\mathbb{C}}=\left[\mathbb{C} P^{n-1}\right] \in$ $H_{2 n-2}\left(\mathbb{C} P^{n} ; \mathbb{Z}_{2}\right)$ and $h_{\mathbb{R}}=\left[\mathbb{R} P^{n-1}\right] \in H_{n-1}\left(\mathbb{R} P^{n} ; \mathbb{Z}_{2}\right)$ the classes of a complex, respectively, real projective hyperplane. These generate the classical intersection products: $h_{\mathbb{C}}^{\cap i}=$ 
$\left[\mathbb{C} P^{2 n-2 i}\right] \in H_{2 n-2 i}\left(\mathbb{C} P^{n} ; \mathbb{Z}_{2}\right)$ and $h_{\mathbb{R}}^{\cap i}=\left[\mathbb{R} P^{n-i}\right] \in H_{n-i}\left(\mathbb{R} P^{n} ; \mathbb{Z}_{2}\right)$. The quantum product on $\mathrm{OH}\left(\mathbb{C} P^{n} ; \Lambda_{X}\right) \cong H\left(\mathbb{C} P^{n} ; \mathbb{Z}_{2}\right) \otimes \Lambda_{X}$ is given by

$$
h_{\mathbb{C}}^{* i}= \begin{cases}h_{\mathbb{C}}^{\cap i}, & i=1, \ldots, n, \\ {\left[\mathbb{C} P^{n}\right] q,} & i=n+1,\end{cases}
$$

see, for example, [22]. It follows that the quantum ring structure on $\mathrm{OH}\left(\mathbb{R} P^{n} ; \Lambda_{R}\right) \cong$ $H\left(\mathbb{R} P^{n} ; \mathbb{Z}_{2}\right) \otimes \Lambda_{R}$ is given by

$$
h_{\mathbb{R}}^{* i}= \begin{cases}h_{\mathbb{R}}^{\cap i}, & i=1, \ldots, n, \\ {\left[\mathbb{R} P^{n}\right] t,} & i=n+1 .\end{cases}
$$

Example 1.2. Consider now $X=\mathbb{C} P^{1} \times \mathbb{C} P^{1} \cong S^{2} \times S^{2}$ with the monotone symplectic form $\omega_{\mathbb{C} P^{1}} \times \omega_{\mathbb{C} P^{1}}$. The real part is $R=\mathbb{R} P^{1} \times \mathbb{R} P^{1} \cong T^{2}$ and we have $C_{X}=N_{R}=2$. Setting $A:=\left[\mathbb{C} P^{1} \times \mathrm{pt}\right], B:=\left[\mathrm{pt} \times \mathbb{C} P^{1}\right] \in H_{2}\left(X ; \mathbb{Z}_{2}\right)$, the quantum product on $\mathrm{QH}\left(X ; \Lambda_{X}\right)=$ $H\left(X ; \mathbb{Z}_{2}\right) \otimes \Lambda_{X}$ satisfies

$$
A * B=B * A=[\mathrm{pt}], \quad A * A=B * B=[X] q .
$$

(In fact, this determines all other products by the associativity of $*$.) Setting $a:=\left[\mathbb{R} P^{1} \times\right.$ $\mathrm{pt}], b:=\left[\mathrm{pt} \times \mathbb{R} P^{1}\right] \in H_{1}\left(R ; \mathbb{Z}_{2}\right)$, we conclude that the quantum product on $\mathrm{OH}\left(R ; \Lambda_{R}\right) \cong$ $H\left(R ; \mathbb{Z}_{2}\right) \otimes \Lambda_{R}$ is determined by

$$
a * b=b * a=[\mathrm{pt}], \quad a * a=b * b=[R] t .
$$

We now give a heuristic explanation for why the wideness part of Theorem A should hold. The quantum homology $\mathrm{OH}\left(R ; \Lambda_{R}\right)$ is the homology of a deformed Morse complex, whose differential $d$ decomposes as

$$
d=d_{0}+d_{1}
$$

The "classical" part $d_{0}$ counts Morse trajectories, while the "quantum" part $d_{1}$ counts pearly trajectories, that is, certain configurations of Morse trajectories with (pseudo)holomorphic discs interspersed between them. Whenever we work with a $\tau$-antiinvariant almost complex structure, such as the standard integrable $J_{0}$, there exists 
an involution on the moduli spaces of discs, given by reflecting discs along $R$, and consequently also on the moduli spaces of pearly trajectories. The idea is that all the quantum contributions entering $d_{1}$ should cancel in the $\mathbb{Z}_{2}$-count because of this involution, so that $d_{1}$ vanishes and hence $d$ is equal to the Morse differential. However, this reasoning does not take into account that the involution might have fixed points (it actually does!), which a priori destroys the argument. Showing that it still works requires to take a closer look at the involution, see Section 6.

To approach the relation between $\mathrm{OH}\left(R ; \Lambda_{R}\right)$ and $\mathrm{QH}\left(X ; \Lambda_{X}\right)$, the first thing to note is that on the classical level there exists a degree-doubling isomorphism

$$
H\left(R ; \mathbb{Z}_{2}\right) \rightarrow H\left(X ; \mathbb{Z}_{2}\right)
$$

which respects the ring structures given by the intersection products. The inverse map is easy to describe: it is induced by mapping all $\tau$-invariant cycles, which generate $H\left(X ; \mathbb{Z}_{2}\right)$, to their $\tau$-fixed parts. We will use an argument of Duistermaat, ultimately based on classical Smith theory, to show that this leads to an isomorphism. While it seems unlikely that this isomorphism has not been known before, the author is not aware of a position in the literature where it appears.

Part (ii) of Theorem A states that this relation stays true on the quantum level. The heuristic idea is similar to the one for the proof of the wideness statement. Since $\mathrm{OH}\left(X ; \Lambda_{X}\right)$ is by definition $H\left(X ; \mathbb{Z}_{2}\right) \otimes \Lambda_{X}$ as a $\Lambda_{X}$-module, the wideness of $R$ allows to conclude immediately that $\mathrm{OH}\left(R ; \Lambda_{R}\right)$ and $\mathrm{OH}\left(X ; \Lambda_{X}\right)$ are isomorphic as modules. In the case of the ambient manifold $X$, the quantum product is a deformation of the classical intersection product using three-point genus zero Gromov-Witten invariants. In the Morse picture, this means that the nonclassical part counts Y-type configurations of Morse trajectories connected by a holomorphic sphere in the center. In the case of the real part $R$, the quantum product counts Y-type configurations of pearly trajectories connected by a holomorphic disc. Similar to the case of the differential, there exists an involution on the moduli space of Y-pearls, and the same type of cancellation argument says that all contributions with a noncentral nonconstant disc should occur in pairs and hence cancel when $\mathbb{Z}_{2}$-counting them. Moreover, if we work with $\tau$-invariant Morse functions $f: X \rightarrow \mathbb{R}$ having all their critical points on $R$, there should be a 1-1 correspondence between the remaining Y-pearls on the Lagrangian side and those on the ambient side.

Again, this argument fails a priori because the involution on the moduli space of discs has fixed points. 


\subsection{Previous results}

The first results in the direction of Theorem A were obtained by Oh [24] who proved that $\operatorname{HF}\left(\mathbb{R} P^{n} ; \mathbb{Z}_{2}\right)$ is isomorphic as a vector space to $H\left(\mathbb{R} P^{n} ; \mathbb{Z}_{2}\right)$. Later Biran and Cornea computed the ring structure of $\mathrm{QH}\left(\mathbb{R} P^{n} ; \Lambda\right)$, in fact, as a special case of a series of more general results for Lagrangians $L \subset \mathbb{C} P^{n}$ satisfying the condition $2 H_{1}(L ; \mathbb{Z})=0$ (see [8] or [6, Section 6.2.2]).

As for general toric manifolds, there is another family of natural Langrangian submanifolds, namely the smooth fibres of the moment map. The study of the Floer homology of the monotone ones was started by Cho and Oh [10] and later extended in a series of papers by Cho. There are also recent results due to Alston and Amorim [2] and Abreu and Macarini [1] on the Floer homology $\operatorname{HF}(R, L)$ of the real part $R$ and a smooth moment fibre $L$.

There have also been efforts to calculate the Floer homology (as a module) of Lagrangians which are fixed point sets of anti-symplectic involutions in more general contexts than that of toric manifolds, see, for example, the works by Frauenfelder [15] and Fukaya et al. [16].

\subsection{Organization of the paper}

We provide the necessary background on symplectic toric manifolds in Section 2. In Section 3, we examine the topology of $R$ and its relation to that of $X$ using $\tau$-invariant Morse functions. More precisely, we prove $H\left(R ; \mathbb{Z}_{2}\right) \cong H\left(X ; \mathbb{Z}_{2}\right)$ as rings. Section 4 provides a sort of classification of the holomorphic discs $u:\left(D^{2}, \partial D^{2}\right) \rightarrow(X, R)$ with boundary on $R$. We show that after removing a boundary point, they admit a representation in toric homogeneous coordinates. This is used in Section 5 to prove that the standard complex structure $J_{0}$ is regular for them. Basically, the same argument will also show the regularity of $J_{0}$ for all holomorphic spheres $v: \mathbb{C} P^{1} \rightarrow X$. In Section 6 , we take a closer look at the involution on the moduli space of real discs. We describe its fixed point set and show that all fixed points we eventually have to count in the definition of the quantum invariants occur in pairs. Section 7 is technical and serves to show that generic choices of $\tau$-invariant data lead to well-behaved moduli spaces. In Section 8, we finally assemble all the ingredients and prove the main result.

\section{Symplectic Toric Manifolds}

There are essentially two ways of constructing a symplectic toric manifold, both starting from a suitable polytope $\Delta$ in $\left(\mathbb{R}^{n}\right)^{*}$. The first is by symplectic reduction of some $\mathbb{C}^{N}$ 
with respect to a torus action, in a way encoded by $\Delta$. In the second, one starts by building a complex manifold $X_{\Sigma}$ together with a torus action from the normal fan $\Sigma$ of $\Delta$, and then embeds $X_{\Sigma}$ into some big projective space to define the symplectic form. In both cases, one obtains a symplectic manifold together with a Hamiltonian torus action whose moment polytope is $\Delta$.

The two constructions are equivalent in the sense that there exist equivariant symplectomorphisms between the resulting symplectic toric manifolds, which follows from a theorem of Delzant [12]. We will describe the second variant as it is more suited to our needs.

The entire section is heavily inspired by the texts from which the author first learned about symplectic toric manifolds, mainly the book by Audin [3] and the lecture notes by Cannas da Silva contained in [4], but also to some extent the treatment in Cho and Oh's paper [10].

\subsection{Cones, fans, and polytopes}

We start by giving definitions of the relevant combinatorial objects.

Definition 2.1. A cone in $\mathbb{R}^{n}$ of dimension $r$ is a subset $\sigma \subset \mathbb{R}^{n}$ that can be written in the form

$$
\sigma=\left\{a_{1} v_{1}+\cdots+a_{r} v_{r} \mid a_{1}, \ldots, a_{r} \geq 0\right\}
$$

with linearly independent vectors $v_{1}, \ldots, v_{r} \in \mathbb{R}^{n}$, the generators of $\sigma$. A cone is called rational and smooth if it admits a set of generators in $\mathbb{Z}^{n}$ which can be extended to a $\mathbb{Z}$-basis of $\mathbb{Z}^{n}$. Any other cone $\sigma^{\prime} \subset \mathbb{R}^{n}$ admitting a set of generators $v_{1}^{\prime}, \ldots, v_{s}^{\prime}$ such that $\left\{v_{1}^{\prime}, \ldots, v_{s}^{\prime}\right\} \subset\left\{v_{1}, \ldots, v_{r}\right\}$ is called a face of $\sigma$.

Definition 2.2. A fan in $\mathbb{R}^{n}$ is a finite set $\Sigma=\left\{\sigma_{1}, \ldots, \sigma_{\ell}\right\}$ of cones in $\mathbb{R}^{n}$ with the following properties:

(i) For every cone $\sigma \in \Sigma$ and every face $\sigma^{\prime}$ of $\sigma$, we have $\sigma^{\prime} \in \Sigma$.

(ii) For all cones $\sigma, \sigma^{\prime} \in \Sigma$, the cone $\sigma \cap \sigma^{\prime}$ is a face of both $\sigma$ and $\sigma^{\prime}$.

$\Sigma$ is complete if in addition the condition

(iii) $\sigma_{1} \cup \cdots \cup \sigma_{\ell}=\mathbb{R}^{n}$

holds. For a fan $\Sigma$, we denote by $\Sigma^{(r)}$ the set of its $r$-dimensional cones. 
Definition 2.3. A polytope $\Delta$ in $\left(\mathbb{R}^{n}\right)^{*}$ is a bounded subset that can be written as the intersection of a finite number of affine half-spaces, that is,

$$
\Delta=\left\{\varphi \in\left(\mathbb{R}^{n}\right)^{*} \mid\left\langle\varphi, v_{i}\right\rangle \geq-a_{i} \forall i \in[1, N]\right\}
$$

with $v_{1}, \ldots, v_{N} \in \mathbb{R}^{n}$ and $a_{1}, \ldots, a_{N} \in \mathbb{R}$. Given a fixed collection of such defining normal vectors $v_{i}$, for $I \subset[1, N]$, the subset

$$
F_{I}:=\left\{\varphi \in \Delta \mid\left\langle\varphi, v_{i}\right\rangle=-a_{i} \forall i \in I\right\}
$$

is called the Ith face of $\Delta$ if it is nonempty.

A polytope $\Delta$ in $\left(\mathbb{R}^{n}\right)^{*}$ gives rise to a fan $\Sigma(\Delta)$ in $\mathbb{R}^{n}$ as follows:

Definition 2.4. Given a face $F_{I}$ of $\Delta$, let $\sigma_{I}$ be the cone generated by the normal vectors $v_{i}, i \in I$. Then

$$
\Sigma(\Delta):=\left\{\sigma_{I} \mid F_{I} \text { is a face of } \Delta\right\}
$$

is a fan called the normal fan of $\Delta$.

\subsection{Constructing a toric manifold from a fan}

Let $\Sigma$ be a complete fan of smooth rational cones of dimension $n$. In this section we will construct the toric manifold associated to $\Sigma$, which by definition is a compact complex manifold

$$
X \equiv X_{\Sigma}
$$

together with an effective action of an $n$-dimensional complex torus $Q_{\mathbb{C}} \cong T_{\mathbb{C}}^{n}$. We write $T_{\mathbb{C}}^{n}$ for $\left(\mathbb{C}^{*}\right)^{n}$ whenever it plays the role of a group acting on a space.)

The starting point for the construction of $X$ is the standard action of $T_{\mathbb{C}}^{N}$ on $\mathbb{C}^{N}$ given by

$$
\left(t_{1}, \ldots, t_{N}\right) \cdot\left(z_{1}, \ldots, z_{N}\right)=\left(t_{1} z_{1}, \ldots, t_{N} z_{N}\right)
$$

The idea is to use the combinatorics of $\Sigma$ to find a certain subtorus $K_{\mathbb{C}} \subset T_{\mathbb{C}}^{N}$ and a subset $\mathcal{U} \subset \mathbb{C}^{N}$ such that the restriction of the action of $K_{\mathbb{C}}$ to $\mathcal{U}$ is free. 
As for the definition of $K_{\mathbb{C}}$, denote by $\left\{v_{1}, \ldots, v_{N}\right\} \subset \mathbb{Z}^{n}$ the set of primitive integral generators of the one-dimensional cones in $\Sigma$ and consider the homomorphism

$$
\pi: \mathbb{Z}^{N} \rightarrow \mathbb{Z}^{n}, \quad e_{j} \mapsto v_{j} \quad \text { for } j=1, \ldots, N
$$

where $e_{j}=(0, \ldots, 1, \ldots, 0) \in \mathbb{Z}^{N}$ is the $j$ th standard basis vector. Denote by $\mathbb{K}$ the kernel of $\pi$ and define $K_{\mathbb{C}}$ to be the subgroup of $T_{\mathbb{C}}^{N}$ generated by all 1-parameter subgroups $T_{\mathbb{C}}^{1} \rightarrow T_{\mathbb{C}}^{N}$ of the form

$$
\tau \mapsto \tau^{\lambda}:=\left(\tau^{\lambda_{1}}, \ldots, \tau^{\lambda_{N}}\right)
$$

with $\lambda=\left(\lambda_{1}, \ldots, \lambda_{N}\right) \in \mathbb{K}$.

Next we construct $\mathcal{U} \subset \mathbb{C}^{N}$. Given a subset $I=\left\{i_{1}, \ldots, i_{r}\right\} \subseteq[1, N]$, consider the linear subspace $Z_{I}:=\left\{z \in \mathbb{C}^{N} \mid i \in I \Longrightarrow z_{i}=0\right\}$ and denote by $\sigma_{I}$ the cone generated by the $v_{i}$, $i \in I$. Then set

$$
\mathcal{U}:=\mathbb{C}^{N} \backslash \bigcup_{\sigma_{I} \notin \Sigma} Z_{I} .
$$

To describe this in words, a point $z=\left(z_{1}, \ldots, z_{N}\right) \in \mathbb{C}^{N}$ belongs to $\mathcal{U}$ iff the cone $\sigma_{I_{z}}$ belongs to $\Sigma$, where $I_{z}:=\left\{i \in[1, N] \mid z_{i}=0\right\}$.

Lemma 2.5. The restriction of the standard action of the subgroup $K_{\mathbb{C}} \subset T_{\mathbb{C}}^{N}$ to the set $\mathcal{U} \subset \mathbb{C}^{N}$ is free.

It follows that the quotient

$$
X \equiv X_{\Sigma}:=\mathcal{U} / K_{\mathbb{C}}
$$

is a complex manifold of dimension $n$, with the complex structure $J_{0}$ inherited from the one on $\mathbb{C}^{N}$. We denote by $\pi: \mathcal{U} \rightarrow X$ the canonical projection and commonly refer to points of $X$ using "toric homogeneous coordinates", that is, we write

$$
\left[z_{1}: \cdots: z_{N}\right]_{X}:=\pi\left(z_{1}, \ldots, z_{N}\right)
$$

in reminiscence of the homogeneous coordinates on projective space. The completeness of $\Sigma$ implies that $X$ is compact (see [3, Proposition VII.1.4]). Moreover, the $T_{\mathbb{C}}^{N}$-action on 
$\mathcal{U}$ induces an action of the quotient

$$
Q_{\mathbb{C}}:=T_{\mathbb{C}}^{N} / K_{\mathbb{C}}
$$

on $X$, which is an $n$-dimensional complex torus.

\subsection{The toric divisors, the first Chern class, and the homology ring}

To any cone $\sigma \in \Sigma$ of dimension $r$, we associate a complex submanifold of $X$ by setting

$$
C_{\sigma}:=\pi\left(Z_{I_{\sigma}}\right)
$$

where $I_{\sigma}:=\left\{i \in[1, N] \mid v_{i}\right.$ generates an edge of $\left.\sigma\right\} . C_{\sigma}$ has complex codimension $r$ and is invariant under the $Q_{\mathbb{C}}$-action because $Z_{I_{\sigma}}$ is invariant under the standard $T_{\mathbb{C}}^{N}$-action. Using toric homogeneous coordinates, we can write

$$
C_{\sigma}=\left\{\left[z_{1}: \cdots: z_{N}\right]_{X} \mid i \in I_{\sigma} \Longrightarrow z_{i}=0\right\}
$$

Of particular importance are the hypersurfaces corresponding to the onedimensional cones in $\Sigma$. We denote them by

$$
D_{1}, \ldots, D_{N} \subset X
$$

and refer to them as the toric divisors. Their homology classes generate $H_{*}\left(X ; \mathbb{Z}_{2}\right)$ as a ring with respect to the intersection product. More precisely, we have

$$
H_{*}\left(X ; \mathbb{Z}_{2}\right) \cong \mathbb{Z}_{2}\left[\left[D_{1}\right], \ldots,\left[D_{N}\right]\right] /(\mathcal{I}+\mathcal{J})
$$

where $\mathbb{Z}_{2}\left[\left[D_{1}\right], \ldots,\left[D_{N}\right]\right]$ is the polynomial ring in the variables $\left[D_{i}\right]$, and the ideals by which one quotients out are

$$
\left.\mathcal{I}=\left\langle\sum_{i=1}^{N}\left\langle\varepsilon, v_{i}\right\rangle\left[D_{i}\right] \mid \varepsilon \in\left(\mathbb{Z}^{n}\right)^{*}\right\rangle, \quad \mathcal{J}=\left\langle\prod_{i \in I}\left[D_{i}\right]\right| I \subset[1, N] \text { with } \sigma_{I} \notin \Sigma\right\rangle .
$$


Moreover, the union of the $D_{i}$ represents the first Chern class of $X$, in the sense that

$$
c_{1}(X)=\mathrm{PD}\left(\left[D_{1}\right]+\cdots+\left[D_{N}\right]\right)
$$

For all of this consult [3].

\subsection{Affine open subsets}

We next introduce a cover of $X$ by open subsets biholomorphic to $\mathbb{C}^{n}$ corresponding to the $n$-dimensional cones in $\Sigma$. Given any such cone $\sigma \in \Sigma^{(n)}$, let $I_{\sigma}=\{i \in[1, N] \mid$ $v_{i}$ generates an edge of $\sigma$ \} be as before and set

$$
V_{\sigma}:=\left\{\left[z_{1}: \cdots: z_{N}\right]_{X} \in X \mid z_{i}=0 \Longrightarrow i \in I_{\sigma}\right\}
$$

That is, $V_{\sigma}$ is obtained from $X$ by cutting out those toric divisors $D_{i}$ for which $v_{i}$ is not part of the set of generators of $\sigma$.

It is easy to see that the $V_{\sigma}$ with $\sigma \in \Sigma^{(n)}$ provide a cover of $X$. Indeed, given any point $z \in \mathcal{U}$, let $I_{z}:=\left\{i \in[1, N] \mid z_{i}=0\right\}$ and note that we have $\sigma_{I_{z}} \in \Sigma$ by the definition of $\mathcal{U}$. Hence $\sigma_{I_{z}}$ is a face of an $n$-dimensional cone $\sigma \in \Sigma$ by the completeness of $\Sigma$, and clearly we have $z \in V_{\sigma}$.

Lemma 2.6. For every $\sigma \in \Sigma^{(n)}$, the set $V_{\sigma} \subset X$ is biholomorphic to $\mathbb{C}^{n}$.

Proof. We assume without loss of generality that the generators of the edges of $\sigma$ are the vectors $v_{k+1}, \ldots, v_{N} \in \mathbb{Z}^{n}$, where $k:=N-n$. These form a basis of $\mathbb{Z}^{n}$, and we denote by $v_{k+1}, \ldots, v_{N} \in\left(\mathbb{Z}^{n}\right)^{*}$ the dual basis.

Next we define a basis $\lambda^{(1)}, \ldots, \lambda^{(k)} \in \mathbb{Z}^{N}$ of $\mathbb{K}=\operatorname{ker} \pi$ by setting

$$
\lambda_{j}^{(i)}:= \begin{cases}\delta_{i j}, & j \in[1, k], \\ -\left\langle v_{j}, v_{i}\right\rangle, & j \in[k+1, N] .\end{cases}
$$

Note that we have $\pi\left(\lambda^{(i)}\right)=v_{i}-\sum_{j=k+1}^{N}\left\langle v_{j}, v_{i}\right\rangle v_{j}$ for all $i \in[1, k]$, and consequently $\left\langle v_{j}, \pi\left(\lambda^{(i)}\right)\right\rangle=\left\langle v_{j}, v_{i}\right\rangle-\left\langle v_{j}, v_{i}\right\rangle=0$ for $j \in[k+1, N]$, which shows that all $\lambda^{(i)}$ belong to $\mathbb{K}$. Since they are clearly linearly independent, they constitute indeed a basis of $\mathbb{K}$.

Let now $z=\left[z_{1}: \cdots: z_{N}\right]_{X} \in V_{\sigma}$, which means that $z_{1}, \ldots, z_{k} \neq 0$. We claim that $z$ is represented by an element of $\mathcal{U}$ of the form $\left(1, \ldots, 1, *_{k+1}, \ldots, *_{N}\right)$. Namely, acting on such 
a vector with $z_{1}^{\lambda^{(1)}} \cdots z_{k}^{\lambda^{(k)}} \in K_{\mathbb{C}}$ yields

$$
z_{1}^{\lambda^{(1)}} \cdot z_{k}^{\lambda^{(k)}} \cdot\left(1, \ldots, 1, *_{k+1}, \ldots, *_{N}\right)=\left(z_{1}, \ldots, z_{k}, *_{k+1}^{\prime}, \ldots, *_{N}^{\prime}\right)
$$

with $*_{j}^{\prime}=*_{j} \cdot \prod_{i=1}^{k} z_{i}^{\lambda_{j}^{(i)}}$ for $j \in[k+1, N]$. Setting $*_{j}:=z_{j} \cdot \prod_{i=1}^{k} z_{i}^{-\lambda_{j}^{(i)}}$ thus produces the required vector. Note that we can write

$$
*_{j}=z_{j} \cdot \prod_{i=1}^{k} z_{i}^{\left\langle v_{j}, v_{i}\right\rangle}=\prod_{i=1}^{N} z_{i}^{\left\langle\nu_{j}, v_{i}\right\rangle},
$$

because $\left\langle v_{j}, v_{i}\right\rangle=\delta_{i j}$ for $i \in[k+1, N]$. It follows that the map given by

$$
\left[z_{1}: \cdots: z_{N}\right]_{X} \mapsto\left(\prod_{i=1}^{N} z_{i}^{\left\langle v_{k+1}, v_{i}\right\rangle}, \ldots, \prod_{i=1}^{N} z_{i}^{\left\langle\nu_{N}, v_{i}\right\rangle}\right)
$$

provides a biholomorphism $V_{\sigma} \cong \mathbb{C}^{n}$.

\subsection{The symplectic form determined by a polytope}

Let $\Delta$ be a polytope in $\left(\mathbb{R}^{n}\right)^{*}$ and let $\Sigma(\Delta)$ be its normal fan. In this section we describe how $\Delta$ determines an embedding of the toric manifold $X \equiv X_{\Sigma(\Delta)}$ into some projective space. Pulling back the Fubini-Study form $\omega_{\mathrm{FS}}$ will then equip $X$ with a symplectic form $\omega$ such that the action of the real torus $Q \subset Q_{\mathbb{C}}$ is Hamiltonian. For this to work we need to assume that $\Delta$ has the following additional properties:

1. $\Delta$ is lattice, in the sense that all vertices belong to $\left(\mathbb{Z}^{n}\right)^{*}$.

2. $\Delta$ is Delzant, meaning that its defining set of normal vectors $v_{i}, i \in[1, N]$, can be chosen such that for every (nonempty) face $F_{I}$, the corresponding collection of $v_{i}, i \in I$, can be extended to a $\mathbb{Z}$-basis of $\left(\mathbb{Z}^{n}\right)^{*}$.

$\Sigma(\Delta)$ is then a complete fan of smooth rational cones, and hence $X$ is a compact toric manifold.

The construction of the embedding goes as follows. Denote by $m_{1}, \ldots, m_{L} \in \Delta \cap$ $\left(\mathbb{Z}^{n}\right)^{*}$ the lattice points of $\Delta$, and let $F_{i} \subset \Delta$ be the $i$ th facet of $\Delta$, that is, the codimension-1 face with normal vector $v_{i}$. Set $\operatorname{LD}\left(m_{i}, F_{j}\right):=\left\langle m_{i}, v_{j}\right\rangle+a_{j} \in \mathbb{Z}$, which is the lattice distance of $m_{i}$ from the facet $F_{j}$. That is, $m_{i}$ lies on the $\operatorname{LD}\left(m_{i}, F_{j}\right)$ th hyperplane parallel to $F_{j}$, 
counting from $F_{j}$. Now define a map $\mathbb{C}^{N} \rightarrow \mathbb{C}^{L}$ by

$$
\left(z_{1}, \ldots, z_{N}\right) \mapsto\left(\prod_{j=1}^{N} z_{j}^{\mathrm{LD}\left(m_{1}, F_{j}\right)}, \ldots, \prod_{j=1}^{N} z_{j}^{\mathrm{LD}\left(m_{L}, F_{j}\right)}\right)
$$

Its restriction to $\mathcal{U} \subset \mathbb{C}^{N}$ takes values in $\mathbb{C}^{L} \backslash\{0\}$ and descends to a map

$$
\Psi: X \rightarrow \mathbb{C} P^{L-1}
$$

which is actually an embedding (see, e.g., [23]). Moreover, it is equivariant with respect to the action of the real torus $Q:=\left\{\left(t_{1}, \ldots, t_{N}\right) \in Q_{\mathbb{C}}|| t_{i} \mid=1 \forall i\right\}$ on $X$ and the $\mathbb{T}^{n}$-action on $\mathbb{C} P^{L-1}$ given by

$$
\left(t_{1}, \ldots, t_{n}\right) \cdot\left[z_{1}: \cdots: z_{L}\right]=\left[t^{m_{1}} z_{1}: \cdots: t^{m_{L}} z_{L}\right]
$$

with $t^{m_{i}}:=t_{1}^{m_{i, 1}} \cdots \cdots t_{n}^{m_{i, n}}$. The latter is Hamiltonian and has moment map

$$
\hat{\mu}: \mathbb{C} P^{L-1} \rightarrow \mathbb{R}^{n}, \quad\left[z_{1}: \cdots: z_{L}\right] \mapsto \frac{\sum_{i=1}^{L} m_{i}\left|z_{i}\right|^{2}}{\sum_{i=1}^{L}\left|z_{i}\right|^{2}}
$$

with respect to (a multiple of) $\omega_{\mathrm{FS}}$. The image of $\hat{\mu}$ is the convex hull of the $m_{i}$, that is, the polytope $\Delta$, and this stays true for the restriction of $\hat{\mu}$ to $\Psi(X)$. Consult the last section of Cannas da Silva's notes [4] for more details on that.

It follows that $\omega:=\Psi^{*} \omega_{\mathrm{FS}}$ makes $X$ a symplectic manifold such that the $Q$-action is Hamiltonian with a moment map $\mu: X \rightarrow \mathfrak{q}^{*}$ whose image is the polytope $\Delta \subset \mathfrak{q}^{*} \cong\left(\mathbb{R}^{n}\right)^{*}$.

\subsection{Complex conjugation}

Complex conjugation on $\mathcal{U} \subset \mathbb{C}^{N}$ descends to an involution

$$
\tau: X \rightarrow X
$$

which we also refer to as complex conjugation. It is anti-symplectic with respect to $\omega=\Psi^{*} \omega_{\mathrm{FS}}$ and related to the torus action by

$$
\tau \circ q=q^{-1} \circ \tau
$$


for $q \in Q$. In particular, $\tau$ maps $Q$-orbits to $Q$-orbits but reverses the time order on them. Observe that $\tau$ leaves the moment map invariant, that is, $\mu \circ \tau=\mu$. This property characterizes $\tau$ among all anti-symplectic involutions on $X$ (up to conjugation by $Q$-equivariant symplectomorphisms).

\section{The Topology of the Real Lagrangian}

Let now $X$ be a symplectic toric manifold of real dimension $2 n$ and $R$ its real part. The purpose of this section is to explain the relation between the homology rings $H_{*}\left(X ; \mathbb{Z}_{2}\right)$ and $H_{*}\left(R ; \mathbb{Z}_{2}\right)$.

\section{1 $\tau$-invariant Morse functions}

Denote by $Q$ the $n$-dimensional torus acting on $X$ in a Hamiltonian way, and by $\mu: X \rightarrow q^{*}$ the corresponding moment map, where $\mathfrak{q}^{*}$ is the dual of the Lie algebra $\mathfrak{q}$ of $Q$. We will work with the following family of $\tau$-invariant functions, defined by

$$
f_{\xi}: X \rightarrow \mathbb{R}, X \mapsto\langle\mu(x), \xi\rangle,
$$

for $\xi \in \mathfrak{q}$. If $\xi$ is chosen generically, then $f_{\xi}$ is Morse, and in this case the critical points of $f_{\xi}$ are precisely the points where $D \mu$ vanishes, that is, the preimages of the vertices of the moment polytope $\Delta$. The Morse index $|p|$ at such a point is given by twice the number of edges of $\Delta$ meeting at the vertex $\mu(p)$ for which the evaluation of an inwardpointing generating vector on $\xi$ is negative. In particular, all critical points have even Morse index, so that $f_{\xi}$ is perfect, that is, the Morse differential vanishes.

The restriction $\left.f_{\xi}\right|_{R}$ is a Morse function on $R$, and the $\tau$-invariance implies easily that all critical points of $\left.f_{\xi}\right|_{R}$ are also critical points of $f_{\xi}$, that is, Crit $\left.f_{\xi}\right|_{R} \subseteq$ Crit $f_{\xi}$. In fact, we even have the following lemma.

Lemma 3.1. The sets Crit $f_{\xi}$ and Crit $\left.f_{\xi}\right|_{R}$ coincide, and the index of any critical point $p$ of $f_{\xi}$ is twice the index of $p$ as a critical point of $\left.f_{\xi}\right|_{R}$.

Proof. For the first statement, it remains to show that Crit $f_{\xi} \subseteq$ Crit $\left.f_{\xi}\right|_{R}$, which clearly follows if we can show Crit $f_{\xi} \subset R$. Note that the $\mu$-preimages of all faces of $\Delta$, in particular of the vertices, are connected and invariant under $\tau$, because their preimages under $\pi: \mathcal{U} \rightarrow X$ are the sets $Z_{I} \subset \mathbb{C}^{N}$ (see Section 2.2), which are connected and invariant under 
complex conjugation. In particular, the preimage of every vertex is a single point in $R$, and hence Crit $f_{\xi}$ is contained in $R$.

The statement about the indices is an immediate consequence of the fact that at every critical point $x$ of $f_{\xi}$, there exist local coordinates $q_{j}, p_{j}$ centered at $x$, such that $f_{\xi}$ can locally be expressed as

$$
f_{\xi}=f_{\xi}(x)+\frac{1}{2} \sum_{j=1}^{n}\left\langle\eta_{j}, \xi\right\rangle\left(q_{j}^{2}+p_{j}^{2}\right)
$$

with certain $\eta_{j} \in \mathfrak{q}^{*}$, and such that $R$ is given by the vanishing of $p$ locally. This is part of Duistermaat [13, Proposition 2.2]. In these coordinates, the Hessians are

$$
\begin{aligned}
\text { Hess }\left.f_{\xi}\right|_{R}(x) & =\operatorname{diag}\left(\left\langle\eta_{1}, \xi\right\rangle, \ldots,\left\langle\eta_{n}, \xi\right\rangle\right), \\
\text { Hess } f_{\xi}(x) & =\operatorname{diag}\left(\left\langle\eta_{1}, \xi\right\rangle, \ldots,\left\langle\eta_{n}, \xi\right\rangle,\left\langle\eta_{1}, \xi\right\rangle, \ldots,\left\langle\eta_{n}, \xi\right\rangle\right)
\end{aligned}
$$

from which the claimed relation follows.

Recall that the definition of Morse homology for a pair $(f, g)$ of a Morse function and a Riemannian metric requires Morse-Smale transversality, meaning that all (un)stable manifolds $W_{x}^{u}(f, g), W_{y}^{s}(f, g)$ intersect transversely. It is a standard fact that for a fixed Morse function, a generic choice of Riemannian metric yields a Morse-Smale pair, see, for example, [20, Proposition 5.8]. In our situation, we are constrained by the fact that we want not only the Morse function, but also the Riemannian metric to be $\tau$-invariant, such as to fully exploit the symmetry. However, the standard result stays true.

Proposition 3.2. Let $f: X \rightarrow \mathbb{R}$ be a $\tau$-invariant Morse function. There exists a second category subset of the space $\mathcal{G}^{\tau}$ of $\tau$-invariant Riemannian metrics on $X$ such that for every $g$ in this subset the pair $(f, g)$ is Morse-Smale.

The proof is almost identical to the proof of the statement without the invariance condition. The only point where a bit of caution is necessary is when local perturbations of the metric along negative gradient flow lines have to be extended to global $\tau$-invariant perturbations. But this poses no problem, basically because if $\gamma$ is such a flow line, we either have $\operatorname{im} \gamma \subset R$ or $\operatorname{im} \gamma \cap \operatorname{im} \tau \circ \gamma=\emptyset$, as a consequence of the fact that both $f$ and $g$ are $\tau$-invariant. (See also Proposition 7.1 in this paper and its proof.) 
Lemma 3.3. Let $f: X \rightarrow \mathbb{R}$ be a $\tau$-invariant Morse function and $g$ a $\tau$-invariant metric on $X$ such that $(f, g)$ is Morse-Smale. Then the restricted pair $\left(\left.f\right|_{R},\left.g\right|_{R}\right)$ is also MorseSmale.

Proof. The invariance implies that $-\nabla f$ is tangent to $R$. Thus, the flow of $-\left.\nabla f\right|_{R}$ is simply the restriction of the flow of $-\nabla f$ to $R$, and the invariant manifolds are related by $W_{X}^{*}\left(\left.f\right|_{R}\right)=W_{X}^{*}(f) \cap R$, for $x \in$ Crit $f \cap R$. Given some $p \in R$, any subspace $V \subset T_{p} X$ with $\tau V=V$ decomposes as $V=V^{+} \oplus V^{-}$, with $V^{ \pm}:=(\operatorname{id} \pm D \tau) V$ denoting its $\tau$-invariant, respectively, $\tau$-anti-invariant subspace, which are both of the same dimension $\frac{1}{2} \operatorname{dim} V$. Now $T_{p} W_{x}^{*}\left(\left.f\right|_{R}\right)=T_{p}\left(W_{x}^{*}(f) \cap R\right)$ is clearly contained in $\left(T_{p} W_{x}^{*}(f)\right)^{+}=T_{p} W_{x}^{*}(f) \cap T_{p} R$, but since both have dimension $\frac{1}{2} \operatorname{dim} W_{x}^{*}(f)$, we actually have $T_{p} W_{x}^{*}\left(\left.f\right|_{R}\right)=\left(T_{p} W_{x}^{*}(f)\right)^{+}$. (In other words, all $W_{X}^{*}(f)$ intersect $R$ cleanly.)

Now $(f, g)$ being Morse-Smale means that $T_{p} W_{x}^{u}(f)+T_{p} W_{y}^{s}(f)=T_{p} X$ at every $p \in$ $W_{x}^{u}(f) \cap W_{y}^{s}(f)$. With the above decomposition, we obtain

$$
\begin{aligned}
T_{p} R & =\left(T_{p} X\right)^{+} \\
& =\left(T_{p} W_{X}^{u}(f)\right)^{+}+\left(T_{p} W_{Y}^{s}(f)\right)^{+} \\
& =T_{p}\left(W_{X}^{u}\left(\left.f\right|_{R}\right)\right)+T_{p}\left(W_{Y}^{s}\left(\left.f\right|_{R}\right)\right),
\end{aligned}
$$

that is, $W_{X}^{u}\left(\left.f\right|_{R}\right)$ and $W_{Y}^{s}\left(\left.f\right|_{R}\right)$, viewed as submanifolds of $R$, intersect transversely at $p$. Hence $\left(\left.f\right|_{R},\left.g\right|_{R}\right)$ is Morse-Smale, too.

\subsection{The $\mathbb{Z}_{2}$-homology ring of $R$}

We now fix a function $f_{\xi}: X \rightarrow \mathbb{R}$ and a $\tau$-invariant Riemannian metric $g$ such that $\left(f_{\xi}, g\right)$ and hence also $\left(\left.f_{\xi}\right|_{R},\left.g\right|_{R}\right)$ are Morse-Smale. In this section we prove the following proposition.

Proposition 3.4. The map Crit $\left.f_{\xi}\right|_{R} \rightarrow$ Crit $f_{\xi}, X \mapsto X$, induces a canonical ring isomorphism

$$
H\left(R ; \mathbb{Z}_{2}\right) \rightarrow H\left(X ; \mathbb{Z}_{2}\right)
$$

which doubles the degree, that is, it takes $H_{*}\left(R ; \mathbb{Z}_{2}\right)$ to $H_{2 *}\left(X ; \mathbb{Z}_{2}\right)$.

This proposition already lets us infer some information about the quantum homology of $R$. Since $H\left(X ; \mathbb{Z}_{2}\right)$ is generated by $H_{2 n-2}\left(X ; \mathbb{Z}_{2}\right)$ as a ring, it shows that 
$H_{*}\left(R ; \mathbb{Z}_{2}\right)$ is generated by $H_{n-1}\left(R ; \mathbb{Z}_{2}\right)$. Under the assumption $N_{R} \geq 2$ on the minimal Maslov number (which we need to assume anyway), this together with [6, Proposition 6.1.1] implies the following corollary.

Corollary 3.5. The $\Lambda_{R}$-wide/narrow dichotomy holds for $R$, that is, $\mathrm{OH}\left(R ; \Lambda_{R}\right)$ either vanishes or is isomorphic to $H\left(R ; \mathbb{Z}_{2}\right) \otimes \Lambda_{R}$ as a $\Lambda_{R}$-module.

The proof of Proposition 3.4 will occupy the rest of this section. We first concentrate on proving that the described map induces an isomorphism of $\mathbb{Z}_{2}$-vector spaces. The main ingredient is the following statement.

Lemma 3.6. The rank of the total homology $H\left(R ; \mathbb{Z}_{2}\right)$ is equal to the number of critical points of $\left.f_{\xi}\right|_{R}$, that is, $\left.f_{\xi}\right|_{R}$ is a perfect Morse function.

This is a special case of a more general result due to Duistermaat [13, Theorem 3.1]. We include the proof for the convenience of the reader, following [13] closely.

Proof of Lemma 3.6. Assume, without loss of generality, that $\xi \in \mathfrak{q}$ is chosen such that $t \mapsto \exp t \xi$ is periodic, which is the case for a dense subset of $\mathfrak{q}$. Furthermore, normalize $\xi$ such that $\exp \xi=1 \in O$.

From the relation between $\tau$ and the torus action, $\tau \circ q=q^{-1} \circ \tau$ for $q \in Q$, we obtain

$$
\tau(\exp (t \xi) \cdot x)=\exp (-t \xi) \cdot x
$$

for $x \in R=$ Fix $\tau$. It follows that the "half-turn map" $\exp \left(\frac{1}{2} \xi\right)=\exp \left(-\frac{1}{2} \xi\right)$ defines an involution of $R$, whose fixed point set we denote by $R_{(1)}$. It is clear that $R_{(1)}$ contains the critical set of $\left.f_{\xi}\right|_{R}$ (the fixed point set of the $Q$-action), but it might be strictly bigger. Therefore, we set more generally

$$
R_{(k)}:=\left\{x \in R_{(k-1)} \mid \exp \left(2^{-k} \xi\right) \cdot x=x\right\}
$$

for $k \geq 1$. This defines a decreasing sequence of submanifolds of $R$ which eventually stabilizes. Since $x \in R_{(\ell)}$ for all $\ell$ implies $d f_{\xi}(x)=0$, we see that in fact we have $R_{(\ell)}=$ Crit $\left.f_{\xi}\right|_{R}$ for all sufficiently large $\ell$.

Now each $R_{(\ell)}$ is the fixed point set of an involution of $R_{(\ell-1)}$. It was shown by Floyd [14] as an application of Smith theory (see, e.g., [9, Section III.3]) that if $M$ is a 
compact manifold and $N$ the fixed point set of a periodic map $M \rightarrow M$ of prime period $p$, then $\operatorname{dim} H\left(M ; \mathbb{Z}_{p}\right) \geq \operatorname{dim} H\left(N ; \mathbb{Z}_{p}\right)$ (the inequality does not necessarily hold in individual degrees). As a consequence, we obtain

$$
\operatorname{dim} H\left(R ; \mathbb{Z}_{2}\right) \geq \operatorname{dim} H\left(R_{(1)} ; \mathbb{Z}_{2}\right) \geq \cdots \geq \operatorname{dim} H\left(\text { Crit }\left.f_{\xi}\right|_{R} ; \mathbb{Z}_{2}\right)
$$

Since the function $\left.f_{\xi}\right|_{R}$ is Morse, the right-hand side is simply the number of its critical points. But this is $\geq \operatorname{dim} H\left(R ; \mathbb{Z}_{2}\right)$, which concludes the proof.

Since we know that $f_{\xi}$ is a perfect Morse function for $X$, this lemma together with Lemma 3.1 allows to conclude that the map described in Proposition 3.4 induces an isomorphism $H\left(R ; \mathbb{Z}_{2}\right) \cong H\left(X ; \mathbb{Z}_{2}\right)$ of vector spaces.

We next argue that the isomorphism preserves the ring structure, that is, is compatible with the intersection product on both sides. Recall first that in order to define the intersection product Morse homologically, one has to work with two different Morse functions $f, f^{\prime}$ and a metric $g$ satisfying a version of Morse-Smale transversality. The product is then induced by the map

$$
\mathbb{Z}_{2}\langle\text { Crit } f\rangle \otimes \mathbb{Z}_{2}\left\langle\text { Crit } f^{\prime}\right\rangle \rightarrow \mathbb{Z}_{2}\langle\text { Crit } f\rangle, \quad x \otimes y \mapsto \sum\langle x * y, z\rangle z,
$$

where the sum runs over all $z \in$ Crit $f^{\prime}$ such that $|x|+|y|-|z|=n$ and the coefficient $\langle x * y, z\rangle \in \mathbb{Z}_{2}$ counts element of the moduli space $\mathcal{Y}\left(x, y, z ; f, f^{\prime}\right)$, whose elements are configurations consisting of a flow line of $-\nabla f$ from $x$ to $z$ which is hit by a flow line of $-\nabla f^{\prime}$ emanating from $y$.

In our particular case, we work again with $\tau$-invariant Morse functions having all their critical points on $R$. While it is tempting to choose $f=f_{\xi}$ and $f^{\prime}=f_{\xi^{\prime}}$ for certain $\xi, \xi^{\prime} \in \mathfrak{q}$, this would be problematic because the critical sets of these coincide, which obstructs the general position requirement. An easy solution to this is to choose instead a small perturbation $f^{\prime}=\tilde{f}_{\xi^{\prime}}$ obtained from $f_{\xi^{\prime}}$ by pushing the critical points slightly off their original position via a $\tau$-equivariant diffeomorphism supported on a small neighborhood of the critical set. An argument similar to the (omitted) proof of Proposition 3.2 shows that Morse-Smale transversality holds for an "even more generic" $\tau$-invariant metric $g$. With these choices, $\tau$ induces an involution

$$
\tau_{*}: \mathcal{Y}\left(x, y, z ; f_{\xi}, \tilde{f}_{\xi^{\prime}}\right) \rightarrow \mathcal{Y}\left(x, y, z ; f_{\xi}, \tilde{f}_{\xi^{\prime}}\right)
$$


on the moduli spaces used to compute the intersection product on $H\left(X ; \mathbb{Z}_{2}\right)$, and the fixed points of $\tau_{*}$ are precisely those configurations which are contained in $R$. All configurations which are not entirely contained in $R$ occur in pairs, so that

$$
\#_{\mathbb{Z}_{2}} \mathcal{Y}\left(x, y, z ; f_{\xi}, \tilde{f}_{\xi^{\prime}}\right)=\#_{\mathbb{Z}_{2}} \mathcal{Y}\left(x, y, z ;\left.f_{\xi}\right|_{R},\left.\tilde{f}_{\xi^{\prime}}\right|_{R}\right)
$$

Because the moduli space on the right-hand side contains precisely those configurations counted for the intersection product on $H\left(R ; \mathbb{Z}_{2}\right)$, this finishes the proof of the statement about the ring structure and hence of Proposition 3.4.

\section{Real Discs and their Doubles}

In this section, we study the holomorphic curves that enter in the definition of the quantum invariants of $R$ and $X$, where holomorphic is always understood with respect to the standard integrable complex structure $J_{0}$.

Definition 4.1. A real disc is a holomorphic map $u:\left(D^{2}, \partial D^{2}\right) \rightarrow(X, R)$, where $D^{2} \subset \mathbb{C}$ denotes the closed unit disc. A real rational curve is a holomorphic map $v: \mathbb{C} P^{1} \rightarrow X$ such that $\tau \circ v \circ C=v$, with $c: \mathbb{C} P^{1} \rightarrow \mathbb{C} P^{1}$ denoting complex conjugation.

The $\tau$-anti-invariance of $J_{0}$ implies that for every real disc $u$, the disc

$$
\bar{u}:\left(D^{2}, \partial D^{2}\right) \rightarrow(X, R), \quad z \mapsto \tau(u(\bar{z})),
$$

obtained by Schwarz reflection is again holomorphic. Gluing the discs $u$ and $\bar{u}$ along their common boundary yields a holomorphic sphere

$$
u^{\sharp}: \mathbb{C} P^{1} \rightarrow X,
$$

which we refer to as the double of $u$. In the usual identification of $D^{2}$ with the extended upper half-plane $\mathbb{H} \cup\{\infty\} \subset \mathbb{C} P^{1}$, the double is given by

$$
u^{\sharp}(z)= \begin{cases}u(z), & z \in \mathbb{H} \cup\{\infty\}, \\ \tau(u(\bar{z})), & \text { otherwise. }\end{cases}
$$


The main point of this section is to provide a representation of real discs and their doubles in toric homogeneous coordinates which will be used for proving the regularity of $J_{0}$ for these curves in Section 5. This strategy is very similar to what Cho and Oh did for discs with boundary on a torus fibre and was actually inspired by their work [10].

\subsection{Lifting real discs and their doubles}

Recall that we constructed the toric manifold $X$ as a quotient of an open set $\mathcal{U} \subset \mathbb{C}^{N}$ by some torus action. It is not difficult to see that any real disc $u$ can be lifted holomorphically to $\mathcal{U}$, that is, that there exists a holomorphic map $w$ such that the diagram

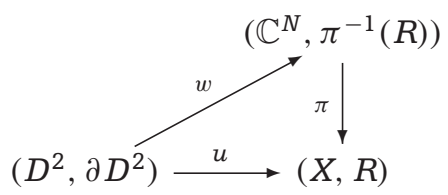

commutes. For the purpose of our transversality proof we would ideally like such a lift to take boundary values in some totally real submanifold of $\mathbb{C}^{N}$ which projects to $R$ under $\pi: \mathcal{U} \rightarrow X$ and whose tangent bundle we understand. In our case the obvious candidate for this submanifold would be $\mathbb{R}^{N}$, but for a nonconstant $u$ it is not possible to find a holomorphic lift satisfying $w\left(\partial D^{2}\right) \subset \mathbb{R}^{N}$ by Liouville's theorem. In this respect, our situation is somewhat different from that considered by Cho and Oh [10], who could lift discs with boundary on a torus fibre such that the boundary was mapped to a totally real torus in $\mathbb{C}^{N}$.

Our solution to this problem is to take out a boundary point and lift only the restriction of $u$ to $\mathbb{H} \subset D^{2}$, or equivalently the restriction of the double $u^{\sharp}$ to $\mathbb{C} \subset \mathbb{C} P^{1}$. It will turn out that this is sufficient for a transversality proof similar to Cho and Oh's to work.

Proposition 4.2. Let $u:\left(D^{2}, \partial D^{2}\right) \rightarrow(X, R)$ be a real disc and let $u^{\sharp}: \mathbb{C} P^{1} \rightarrow X$ be its double. The restriction of $u^{\sharp}$ to $\mathbb{C} \subset \mathbb{C} P^{1}$ admits a lift

$$
w=\left(w_{1}, \ldots, w_{N}\right):(\mathbb{C}, \mathbb{R}) \rightarrow\left(\mathbb{C}^{N}, \mathbb{R}^{N}\right),
$$


where the $w_{i}: \mathbb{C} \rightarrow \mathbb{C}$ are holomorphic functions of the form

$$
w_{i}(z)=a_{i} \cdot \prod_{j=1}^{\alpha_{i}}\left(z-p_{i, j}\right)\left(z-\overline{p_{i, j}}\right) \cdot \prod_{k=1}^{\beta_{i}}\left(z-q_{i, j}\right)
$$

with constants $a_{i} \in \mathbb{R}$, integers $\alpha_{i}, \beta_{i} \geq 0$, and points $p_{i, j} \in \mathbb{C} \backslash \mathbb{R}, q_{i, j} \in \mathbb{R}$.

Proof. We start with an arbitrary holomorphic lift

$$
w=\left(w_{1}, \ldots, w_{N}\right):(\mathbb{C}, \mathbb{R}) \rightarrow\left(\mathbb{C}^{N}, \pi^{-1}(R)\right)
$$

of the double $u^{\sharp}: \mathbb{C} \rightarrow X$, which exists because the pullback of the holomorphic principal $K_{\mathbb{C}}$-bundle $\mathcal{U} \rightarrow X$ to $\mathbb{C}$ by $u^{\sharp}$ is holomorphically trivial (see, e.g., [18]). Denote by $I_{0} \subset$ $[1, N]$ the set of all $i$ such that $u\left(D^{2}\right)$ is contained in the hypersurface $D_{i}$, and set $\overline{I_{0}}:=$ $[1, N] \backslash I_{0}$. For $i \in \overline{I_{0}}, u^{\sharp}$ hits $D_{i}$ at only finitely many points, and hence $w_{i}$ has only finitely many zeros. Clearly, the set of zeros of $w_{i}$ is invariant under complex conjugation by the $\tau$-invariance of $u^{\sharp}$. Denote by $p_{i, 1}, \overline{p_{i, 1}}, \ldots, p_{i, \alpha_{i}}, \overline{p_{i, \alpha_{i}}}$ the zeros of $w_{i}$ in $\mathbb{C} \backslash \mathbb{R}$, and by $q_{i, 1}, \ldots, q_{1, \beta_{i}}$ those in $\mathbb{R}$. In both cases, a zero with multiplicity $m$ is treated as $m$ zeros. Now define a new holomorphic map

$$
w^{\prime}=\left(w_{1}^{\prime}, \ldots, w_{N}^{\prime}\right): \mathbb{C} \rightarrow \mathbb{C}^{N}
$$

by setting

$$
w_{i}^{\prime}(z):=\frac{w_{i}(z)}{\chi_{i}(z)}
$$

with $\chi_{i}(z):=\prod_{j=1}^{\alpha_{i}}\left(z-p_{i, j}\right)\left(z-\overline{p_{i, j}}\right) \cdot \prod_{j=1}^{\beta_{i}}\left(z-q_{i, j}\right)$. Finally set

$$
u^{\prime}:=\pi \circ w^{\prime}: \mathbb{C} \rightarrow X
$$

Claim. $\quad u^{\prime}: \mathbb{C} \rightarrow X$ is constant with image in $R$.

It is easy to see that $u^{\prime}$ extends over $\infty$ to a holomorphic map $u^{\prime}: \mathbb{C} P^{1} \rightarrow X$, using, for example, the projective embedding $\Psi: X \rightarrow \mathbb{C} P^{L}$ described in Section 2. Note that $\infty$ is the only point where this extension can possibly meet any of the hypersurfaces $D_{i}$, $i \in \overline{I_{0}}$, because for those $i$ the corresponding $w_{i}^{\prime}: \mathbb{C} \rightarrow \mathbb{C}$ are nowhere-vanishing. It follows that all the $D_{i}$ with $u^{\prime}\left(\mathbb{C} P^{1}\right) \cap D_{i} \neq \emptyset$, including those with $i \in I_{0}$, intersect at $u^{\prime}(\infty)$. In 
particular, this intersection is nonempty, and thus the cone $\sigma$ generated by the corresponding normal vectors $v_{i}$ of $\Delta$ is contained in the normal fan of $\Delta$. Let $\sigma^{\prime} \in \Sigma^{(n)}$ be an $n$-dimensional cone containing $\sigma$ as a face. Hence $u^{\prime}\left(\mathbb{C} P^{1}\right)$ is entirely contained in the set $V_{\sigma^{\prime}}$, which by Lemma 2.6 is biholomorphic to $\mathbb{C}^{n}$. So $u^{\prime}$ is constant by Liouville's theorem, and the fact that all $\chi_{j}$ send real numbers to real numbers implies that the image of $u^{\prime}$ actually lies in $R$. This proves the claim.

To conclude the proof of the proposition, note that we may assume that also the lift $w^{\prime}$ of $u^{\prime}$ is constant (otherwise $w^{\prime}$ is of the form $w^{\prime}(z)=w^{\prime \prime}(z) \cdot w^{\prime}(0)$ with $w^{\prime \prime}$ taking values in $K_{\mathbb{C}}$, in which case we can replace the original $w$ by $\left.\left(w^{\prime \prime}\right)^{-1} \cdot w\right)$, and consequently the image of $w^{\prime}$ is a point in $\pi^{-1}(R)$. By eventually acting with some element of $K_{\mathbb{C}}$, we may further assume that this point actually lies in $\mathbb{R}^{N}$. Recalling the way $w^{\prime}$ and $w$ are related shows that $w$ is of the claimed form.

\subsection{Reading off the Maslov index}

As for the relationship between the Maslov index $\mu(u)$ of a real disc and the first Chern class $C_{1}\left(u^{\sharp}\right)$ of its double, we have

$$
\begin{aligned}
c_{1}\left(u^{\sharp}\right) & =\frac{1}{2}(\mu(u)+\mu(\bar{u})) \\
& =\mu(u) .
\end{aligned}
$$

The first equality is well known, and the second follows from $\mu(u)=\mu(\bar{u})$. Given that $c_{1}(X)$ is Poincaré dual to $\left[D_{1}\right]+\cdots+\left[D_{N}\right]$, we conclude that the Maslov index of $u$ is equal to the intersection number of $u^{\sharp}$ with the toric divisors. In case $u$ is not entirely contained in any $D_{i}$ and hence intersects each of them only a finite number of times, this number is simply

$$
\mu(u)=\sum_{i=1}^{N} \#\left\{\text { zeros of } w_{i}\right\}
$$

where the $w_{i}$ are the components of any holomorphic lift $w: \mathbb{C} \rightarrow \mathbb{C}^{N}$ of $u^{\sharp}$, counting with multiplicities. We assume here that $u(\infty)$ is not contained in any $D_{i}$, so that all intersection points of $u$ with the $D_{i}$ correspond to zeros of the $w_{i}$.

If $u$ is entirely contained in some $D_{i}$, or equivalently $w_{i}$ vanishes identically, one has to represent $\left[D_{i}\right]$ by a linear combination of those $\left[D_{j}\right]$ for which $u$ is not contained in $D_{j}$ in order to determine the intersection number of $u^{\sharp}$ with $\left[D_{i}\right]$. 
This results in the following general recipe for writing the Maslov index in terms of a weighted count of the numbers of isolated zeros of the components of $w$. As in the proof of Proposition 4.2 we denote by $I_{0}=\left\{i_{1}, \ldots, i_{\ell}\right\}$ the set of those $i \in[1, N]$ such that $u\left(D^{2}\right)$ is contained in $D_{i}$ and set $\overline{I_{0}}:=[1, N] \backslash I_{0}$. Moreover, we assume that $u(\infty) \notin$ $\bigcup_{i \in \bar{I}_{0}} D_{i}$, which can be arranged using a suitable reparametrization. Observe now that $\bigcap_{i \in I_{0}} D_{i} \neq \emptyset$ because $u\left(D^{2}\right)$ is contained in the intersection. It follows that the $v_{i_{1}}, \ldots, v_{i_{\ell}}$ generate a cone in $\Sigma$, and so they can be extended to a $\mathbb{Z}$-basis of $\mathbb{Z}^{n}$. Fixing any such basis, we denote by $\varepsilon_{i_{1}}, \ldots, \varepsilon_{i_{\ell}} \in\left(\mathbb{Z}^{n}\right)^{*}$ those elements of the corresponding dual basis of $\left(\mathbb{Z}^{n}\right)^{*}$ which are dual to the $v_{i_{1}}, \ldots, v_{i_{\ell}}$, that is, $\left\langle\varepsilon_{i_{j}}, v_{i_{k}}\right\rangle=\delta_{j k}$ for $j, k=1, \ldots, \ell$.

Proposition 4.3. The Maslov index of $u$ can be expressed as

$$
\mu(u)=\sum_{j \in \overline{I_{0}}}\left(1-\sum_{i \in I_{0}}\left\langle\varepsilon_{i}, v_{j}\right\rangle\right)\left(2 \alpha_{j}+\beta_{j}\right),
$$

where $\alpha_{i}$ and $\beta_{i}$ are as in Proposition 4.2.

Proof. By the description of $H\left(X ; \mathbb{Z}_{2}\right)$ as given in Section 2, the identity

$$
\sum_{j=1}^{N}\left\langle\varepsilon_{i}, v_{j}\right\rangle\left[D_{j}\right]=0
$$

in $H_{2 n-2}\left(X ; \mathbb{Z}_{2}\right)$ holds for all $i \in I_{0}$. The choice of the $\varepsilon_{i}$ implies that

$$
\left[D_{i}\right]=-\sum_{j \in \overline{I_{0}}}\left\langle\varepsilon_{i}, v_{j}\right\rangle\left[D_{j}\right]
$$

for all $i \in I_{0}$. Since $C_{1}(X)$ is Poincaré dual to $\left[D_{1}\right]+\cdots+\left[D_{N}\right]$, we obtain

$$
\begin{aligned}
C_{1}(X) & =\mathrm{PD}\left(\sum_{i \in I_{0}}\left(-\sum_{j \in \overline{I_{0}}}\left\langle\varepsilon_{i}, v_{j}\right\rangle\left[D_{j}\right]\right)+\sum_{j \in \overline{I_{0}}}\left[D_{j}\right]\right) \\
& =\operatorname{PD}\left(\sum_{j \in \overline{I_{0}}}\left(1-\sum_{i \in I_{0}}\left\langle\varepsilon_{i}, v_{j}\right\rangle\right)\left[D_{j}\right]\right),
\end{aligned}
$$

which implies the formula claimed for $\mu(u)$. 
Example. We include a baby example to illustrate how reading off the Maslov index works. Consider the fan $\Sigma$ in $\mathbb{R}^{2}$ whose one-dimensional cones are generated by

$$
v_{1}=(1,0), \quad v_{2}=(0,1), \quad v_{3}=(-1,-1), \quad v_{4}=(0,-1) .
$$

The corresponding toric manifold is $X=\mathbb{C} P^{2} \# \overline{\mathbb{C} P^{2}}$, a toric one-point blow-up of $\mathbb{C} P^{2}$. Observe that we have $\left[z_{1}: z_{2}: z_{3}: z_{4}\right]=\left[t z_{1}, s t z_{2}, t z_{3}, s z_{4}\right]$ for $(s, t) \in T_{\mathbb{C}}^{2}$ because the map $\pi$ : $\mathbb{Z}^{4} \rightarrow \mathbb{Z}^{2}, e_{i} \mapsto v_{i}$, has kernel $\mathbb{K}=\mathbb{Z}\langle(0,1,0,1),(1,1,1,0)\rangle$. Consider now the real rational curve $u$ whose restriction to $\mathbb{H}$ is given by

$$
u(z)=[z: 1: 1: 0]
$$

or equivalently, whose lift to $\mathbb{C}^{2}$ is $w(z)=(z, 1,1,0)$. It sweeps out one half of the divisor $D_{4}$. For $z \neq 0$ we can write $u(z)=\left[1: 1: z^{-1}: 0\right]$ by acting with $\left(1, z^{-1}\right) \in T_{\mathbb{C}}^{2}$, and hence $u$ intersects $D_{3}$ at $\infty$. To move this intersection away from $\infty$, we reparametrize $u$ using the element $\phi \in \operatorname{Aut}\left(D^{2}\right), z \mapsto \frac{z}{z-1}$, such as to obtain the new real disc

$$
u^{\prime}(z)=\left[\frac{z}{z-1}: 1: 1: 0\right]=[z: 1: z-1: 0],
$$

which has the form required for the formula of Proposition 4.3 to be applicable. We have $I_{0}=\{4\}, \alpha_{i}=0$ for all $i, \beta_{1}=\beta_{3}=1$ and $\beta_{2}=0$. Choosing, for example, the vector $(1,1)$ to extend $v_{4}=(0,-1)$ to a basis of $\mathbb{Z}^{2}$, the required element of the dual basis is $\varepsilon_{4}=(1,-1)$, where we identify $\left(\mathbb{Z}^{2}\right)^{*}$ with $\mathbb{Z}^{2}$ in the usual way. With $\left\langle\varepsilon_{4}, v_{1}\right\rangle=1$ and $\left\langle\varepsilon_{4}, v_{3}\right\rangle=0$ we obtain

$$
\mu(u)=\mu\left(u^{\prime}\right)=\left(1-\left\langle\varepsilon_{4}, v_{1}\right\rangle\right) \beta_{1}+\left(1-\left\langle\varepsilon_{4}, v_{3}\right\rangle\right) \beta_{3}=1
$$

as expected, because $D_{4}$ is the exceptional divisor. (In particular, we have $C_{X}=1$, so that our main theorem is not applicable to $X$.)

\section{Regularity of the Standard Complex Structure}

In this section we will prove the following theorem.

Theorem 5.1. The standard complex structure $J_{0}$ is regular for all holomorphic real discs $u:\left(D^{2}, \partial D^{2}\right) \rightarrow(X, R)$ and all holomorphic spheres $v: \mathbb{C} P^{1} \rightarrow X$. 
The proof is similar to Cho and Oh's regularity proof in [10]. We will give full details only for the statement about discs, and then sketch the essentially identical proof for the sphere case in the last subsection.

\subsection{Reformulating the problem}

Recall that Fredholm regularity of $J_{0}$ for $u$ means that the linearization $D_{u} \bar{\partial}_{J_{0}}$ of the operator $\bar{\partial}_{J_{0}}$ at $u$ is surjective. Here we view $\bar{\partial}_{J_{0}}$ as a section in a suitable Banach manifold setting. In our case, this linearization is an operator

$$
D_{u} \bar{\partial}_{J_{0}}: W^{1, p}\left(u^{*} T X, u^{*} T R\right) \rightarrow L^{p}\left(\Lambda^{0,1} T^{*} D \otimes u^{*} T X\right)
$$

for some $p>2$, whose restriction to smooth sections is the standard Dolbeault operator

$$
\bar{\partial}: C^{\infty}\left(u^{*} T X, u^{*} T R\right) \rightarrow \Omega^{0,1}\left(u^{*} T X\right) .
$$

(Here and in the following "smooth" means "smooth in the interior and all derivatives continuous up to the boundar".) Since we know that $D_{u} \bar{\partial}_{J_{0}}$ is Fredholm by general theory and because smooth sections are dense, it suffices to show that $\bar{\partial}$ is surjective, that is,

$$
\text { coker } \bar{\partial}=0 \text {. }
$$

This is equivalent to the vanishing of a certain sheaf cohomology group. To first discuss this in general terms, let $(E, F)$ be a holomorphic bundle pair over $\left(D^{2}, \partial D^{2}\right)$, that is, let $E$ be a holomorphic vector bundle over $D^{2}$ and let $F$ be a totally real subbundle over $\partial D^{2}$. Denote by $\mathcal{O}(E, F)$ the sheaf of holomorphic sections of $E$ taking boundary values in $F$, by $\mathcal{A}^{0}(E, F)$ the sheaf of smooth sections of $E$ taking boundary values in $F$, and by $\mathcal{A}^{(0,1)}(E)$ the sheaf of smooth $E$-valued $(0,1)$-forms. The latter two are fine sheaves, that is, they admit partitions of unity.

Proposition 5.2. The sequence of sheaves $\mathcal{A}^{0}(E, F) \stackrel{\bar{\partial}}{\rightarrow} \mathcal{A}^{(0,1)}(E) \rightarrow 0 \rightarrow \cdots$ is a fine resolution of $\mathcal{O}(E, F)$.

This is proved (for arbitrary Riemann surfaces with boundary), for example, in [21]. It follows that the sheaf cohomology groups of $\mathcal{O}(E, F)$, which are by definition the 
right derived functors of the global section functor applied to $\mathcal{O}(E, F)$, satisfy

$$
H^{*}\left(D^{2} ; \mathcal{O}(E, F)\right)= \begin{cases}\operatorname{ker} \bar{\partial}, & *=0, \\ \operatorname{coker} \bar{\partial}, & *=1, \\ 0, & * \geq 2 .\end{cases}
$$

The idea for our specific problem is to show $H^{1}(D ; \mathcal{O}(E, F))=0$ for $(E, F)=$ $\left(u^{*} T X, u^{*} T R\right.$ ) by exhibiting a suitable acyclic cover of $D^{2}$ and making use of the fact that sheaf cohomology groups can be computed as sheaf-valued Čech cohomology groups with respect to an acyclic cover.

\subsection{An acyclic cover}

By definition, acyclicity of a cover for a sheaf means that all higher cohomology groups ( $H^{i}$ with $i \geq 1$ ) of the sheaf restricted to arbitrary intersections of the covering sets vanish. Consider the cover $\mathfrak{U}=\left\{U_{0}, U_{1}\right\}$ of $D^{2}$ with

$$
U_{i}=D^{2} \backslash\left\{z_{i}\right\}
$$

where $z_{0}=0$ and $z_{1}=\infty$, thinking of $D^{2}$ as the extended upper half-plane. The purpose of this subsection is to prove that $\mathfrak{U}$ is acyclic for $\mathcal{O}(E, F)$, that is, that

$$
H^{1}\left(U_{0} ; \mathcal{O}(E, F)\right)=H^{1}\left(U_{1} ; \mathcal{O}(E, F)\right)=H^{1}\left(U_{0} \cap U_{1} ; \mathcal{O}(E, F)\right)=0
$$

As both $U_{i}$ are biholomorphic to the upper half-plane, in the following we simply write $\mathbb{H}$ to represent either of them. Moreover, we write $\mathbb{H}^{*}$ for $U_{0} \cap U_{1}$.

Let $w:(\mathbb{H}, \mathbb{R}) \rightarrow\left(\mathbb{C}^{N}, \mathbb{R}^{N}\right)$ be a lift of $u_{\mathbb{H}}$, which exists by Proposition 4.2 , and consider the following bundle pairs over $(\mathbb{H}, \mathbb{R})$ :

$$
\begin{aligned}
\left(E_{K}, F_{K}\right) & :=\left(w^{*}\left(\operatorname{TOrb}_{K_{\mathbb{C}}}\right), w^{*}\left(\operatorname{TOrb}_{K_{\mathbb{C}}} \cap \mathbb{R}^{N}\right)\right), \\
(\tilde{E}, \tilde{F}) & :=\left(w^{*} \mathbb{C}^{N}, w^{*} \mathbb{R}^{N}\right), \\
(E, F) & :=\left(u^{*} T X, u^{*} T R\right) .
\end{aligned}
$$

Here $T \operatorname{Orb}_{K_{\mathbb{C}}}$ denotes the subbundle of $T \mathbb{C}^{N}$ whose fibre at $z \in \mathbb{C}^{N}$ is the tangent space

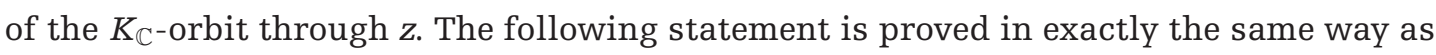
Lemma 6.3 in [10]. 
Lemma 5.3. The natural sequence of sheaves

$$
0 \rightarrow \mathcal{O}\left(E_{K}, F_{K}\right) \rightarrow \mathcal{O}(\tilde{E}, \tilde{F}) \rightarrow \mathcal{O}(E, F) \rightarrow 0
$$

is exact.

This implies that there exists a long exact sequence in cohomology of the form

$$
\begin{aligned}
0 & \rightarrow H^{0}\left(\mathbb{H} ; \mathcal{O}\left(E_{K}, F_{K}\right)\right) \rightarrow H^{0}(\mathbb{H} ; \mathcal{O}(\tilde{E}, \tilde{F})) \rightarrow H^{0}(\mathbb{H} ; \mathcal{O}(E, F)) \\
& \rightarrow H^{1}\left(\mathbb{H} ; \mathcal{O}\left(E_{K}, F_{K}\right)\right) \rightarrow H^{1}(\mathbb{H} ; \mathcal{O}(\tilde{E}, \tilde{F})) \rightarrow H^{1}(\mathbb{H} ; \mathcal{O}(E, F)) \rightarrow 0
\end{aligned}
$$

The sequence stays exact when $\mathbb{H}$ is replaced by the open subset $\mathbb{H}^{*}$. To show $H^{1}(\mathbb{H} ; \mathcal{O}(E, F))=H^{1}\left(\mathbb{H}^{*} ; \mathcal{O}(E, F)\right)=0$ and thus the acyclicity of $\mathfrak{U}$, it is hence sufficient to show

$$
H^{1}(\mathbb{H} ; \mathcal{O}(\tilde{E}, \tilde{F}))=H^{1}\left(\mathbb{H}^{*} ; \mathcal{O}(\tilde{E}, \tilde{F})\right)=0
$$

Since $(\tilde{E}, \tilde{F})=\left(\mathbb{H} \times \mathbb{C}^{N}, \mathbb{R} \times \mathbb{R}^{N}\right)$ is the trivial bundle with a trivial totally real subbundle, this follows from the next proposition.

Proposition 5.4. Given a smooth function $g: \mathbb{H} \rightarrow \mathbb{C}$ such that $g(\mathbb{R}) \subset \mathbb{R}$, there exists a smooth function $f: \mathbb{H} \rightarrow \mathbb{C}$ satisfying $\bar{\partial} f=g$ and $f(\mathbb{R}) \subset \mathbb{R}$.

We include (parts of) the proof for the convenience of the reader, essentially reproducing the proof of Theorem 1.11 in [19].

Proof. For compactly supported functions $g$ the statement is a rather well-known result from complex analysis.

Suppose now that $g$ is not compactly supported. For $n \in \mathbb{N}$, let $B_{n}:=\{z \in \mathbb{H}|| z \mid<n\}$ be the open half-disc of radius $n$, let $\rho_{n}$ be a cutoff function with $\rho_{n} \equiv 1$ on $\overline{B_{n}}$, supp $\rho_{n} \subset$ $B_{n+1}$ and let $g_{n}:=\rho_{n} \cdot g$. We will construct a sequence of smooth functions $f_{n}: \mathbb{H} \rightarrow \mathbb{C}$ such that

(i) $\bar{\partial} f_{n}=g$ in $B_{n}$.

(ii) $f_{n}(\mathbb{R}) \subset \mathbb{R}$.

(iii) $\left|f_{n+1}(z)-f_{n}(z)\right| \leq 2^{-n}$ for all $z \in \overline{B_{n-1}}$. 
Suppose that functions $f_{1}, \ldots, f_{n}$ satisfying these conditions have been constructed. To define $f_{n+1}$, start with a function $\varphi: \mathbb{H} \rightarrow \mathbb{C}$ solving $\bar{\partial} \varphi=g_{n+1}$ and $\varphi(\mathbb{R}) \subset \mathbb{R}$, which exists because $g_{n+1}$ has compact support. The function $\varphi-f_{n}$ is holomorphic on $B_{n}$ and satisfies $\left(\varphi-f_{n}\right)(\mathbb{R}) \subset \mathbb{R}$; by truncating its power series expansion around 0 at a sufficiently high power, we obtain a polynomial $\psi$ which is holomorphic on $B_{n+1}$, satisfies $\psi(\mathbb{R}) \subset \mathbb{R}$, and $\left|\varphi(z)-f_{n}(z)-\psi(z)\right|<2^{-n}$ for all $z \in \overline{B_{n-1}}$. Then we define

$$
f_{n+1}:=\varphi-\psi
$$

and note that it has the required properties.

As for the convergence of this sequence of functions, observe that all $f_{m}$ with $m \geq n$ satisfy $\bar{\partial} f_{m}=g$ on $B_{n}$. We can hence write

$$
f_{m}(z)=f_{n}(z)+\sum_{j=n+1}^{m} f_{j}(z)-f_{j-1}(z)
$$

for $z \in B_{n}$. Since all $f_{j}-f_{j-1}$ in the sum are holomorphic on $B_{n}$ and because of the uniform bounds they satisfy on $\overline{B_{n-1}}$, the sum converges for $m \rightarrow \infty$ to a function which is holomorphic on $B_{n}$ and takes $\mathbb{R}$ to $\mathbb{R}$. Since this is true for every $n$, it follows that the $f_{m}$ converge for $m \rightarrow \infty$, uniformly on compact sets, to a function $f: \mathbb{H} \rightarrow \mathbb{C}$ satisfying $\bar{\partial} f=g$ and $f(\mathbb{R}) \subset \mathbb{R}$.

\subsection{A Čech cohomology computation}

In this section we finish the proof of

$$
H^{1}\left(D^{2} ; \mathcal{O}(E, F)\right)=0
$$

and hence of Theorem 5.1. We make use of the following classical theorem:

Theorem 5.5 (Leray). Let $X$ be a topological space, $\mathcal{S}$ a sheaf on $X$, and let $\mathfrak{U}=\left\{U_{i}\right\}_{i \in I}$ be a countable cover of $X$ which is acyclic with respect to $\mathcal{S}$. Then

$$
H^{*}(X ; \mathcal{S}) \cong \check{H}^{*}(\mathfrak{U} ; \mathcal{S})
$$

Here $H^{*}(X ; \mathcal{S})$ denotes the sheaf cohomology of $\mathcal{S}$ in the sense of "right derived functor of the global section functor", and $\check{H}^{*}(\mathfrak{U} ; \mathcal{S})$ denotes Čech cohomology with 
respect to the cover $\mathfrak{U}$ with values in $\mathcal{S}$. So we are done if we can show

$$
\check{H}^{1}(\mathfrak{U} ; \mathcal{O}(E, F))=0
$$

for the acyclic cover $\mathfrak{U}=\left\{U_{0}, U_{1}\right\}$ from the previous subsection. That is, we have to show that every Čech 1-cocycle is a coboundary. Since our cover consists of only two sets, a 1-cocycle is simply a section $\sigma$ of $\mathcal{O}(E, F)$ over $U_{0} \cap U_{1}=\mathbb{H}^{*}$, and for $\sigma$ to be a coboundary means that there exist sections $\eta_{0}, \eta_{1}$ over $U_{0}, U_{1}$ such that

$$
\sigma=\left.\eta_{0}\right|_{U_{0} \cap U_{1}}+\left.\eta_{1}\right|_{U_{0} \cap U_{1}} .
$$

In other words, all we have to show is the following claim.

Claim. Any section $\sigma$ of $\mathcal{O}(E, F)$ over $\mathbb{H}^{*}$ decomposes as

$$
\sigma=\eta_{0}+\eta_{1}
$$

with sections $\eta_{i}$ over $\mathbb{H}^{*}$ such that $\eta_{0}$ extends over 0 and $\eta_{1}$ extends over $\infty$.

Proof of the Claim. Step 1. Fix a lift $w:(\mathbb{H}, \mathbb{R}) \rightarrow\left(\mathbb{C}^{N}, \mathbb{R}^{N}\right)$ of the restriction of $u$ to $U_{0}=\mathbb{H}$, and denote by $w$ also the restriction to $\mathbb{H}^{*}$. Let $\left(E_{K}, F_{K}\right),(\tilde{E}, \tilde{F}),(E, F)$ be the corresponding bundle pairs over $\mathbb{H}, \mathbb{H}^{*}$ as in the last subsection. We claim that $\sigma$ lifts to a section $\tilde{\sigma}$ of the trivial bundle pair $(\tilde{E}, \tilde{F})$ over $\mathbb{H}^{*}$. In other words, we claim the map

$$
H^{0}\left(\mathbb{H}^{*} ; \mathcal{O}(\tilde{E}, \tilde{F})\right) \rightarrow H^{0}\left(\mathbb{H}^{*} ; \mathcal{O}(E, F)\right)
$$

induced by the sequence of sheaves in Lemma 5.3 is surjective, which in view of the long exact sequence is equivalent to saying that

$$
H^{1}\left(\mathbb{H}^{*} ; \mathcal{O}\left(E_{K}, F_{K}\right)\right)=0
$$

That this is indeed the case follows from the next lemma and Proposition 5.4 (which also holds when $\mathbb{H}$ is replaced by $\mathbb{H}^{*}$ ).

Lemma 5.6. The bundle pair $\left(E_{K}, F_{K}\right)=\left(w^{*}\left(\operatorname{TOrb}_{K_{\mathbb{C}}}\right), w^{*}\left(\operatorname{TOrb}_{K_{\mathbb{C}}} \cap \mathbb{R}^{N}\right)\right)$ is holomorphically trivial. 
Proof. Let $z \in \mathcal{U} \subset \mathbb{C}^{N}$. Then we have

$$
E_{K, Z}=T_{Z} O r b_{K_{\mathbb{C}}}=z \cdot T_{1} O r b_{K_{\mathbb{C}}}
$$

where the right-hand side denotes the subspace of $T_{z} \mathbb{C}^{N} \cong \mathbb{C}^{N}$ obtained by letting $z$ act on the subspace $T_{\underline{1}} K_{\mathbb{C}}$ of $T_{1} \mathbb{C}^{N} \cong \mathbb{C}^{N}$ in the usual way (i.e., $z \cdot\left(t_{1}, \ldots, t_{N}\right)=\left(z_{1} t_{1}, \ldots, z_{N} t_{N}\right)$ ), and $\underline{1}:=(1, \ldots, 1) \in \mathbb{C}^{N}$. This follows directly from the fact that $K_{\mathbb{C}}$ acts freely on $\mathcal{U}$, which is equivalent to saying that the map $K_{\mathbb{C}} \ni t \mapsto z \cdot t$ is a diffeomorphism onto its image. For $z \in \mathcal{U} \cap \mathbb{R}^{N}$ the same argument shows

$$
F_{K, z}=\left(T_{Z} O r b_{K_{\mathbb{C}}}\right) \cap \mathbb{R}^{N}=z \cdot\left(T_{\underline{1}} \operatorname{Orb}_{K_{\mathbb{C}}} \cap \mathbb{R}^{N}\right)
$$

It follows that

$$
\begin{aligned}
\mathbb{H} \times T_{\underline{1}} \operatorname{Orb}_{K_{\mathbb{C}}} & \rightarrow E_{K}, \\
(z, t) & \mapsto(z, w(z) \cdot t)
\end{aligned}
$$

is a holomorphic trivialization of $E_{K}$ identifying $F_{K}$ with a constant totally real subbundle.

Proof of the Claim, Step 2. Since $(\tilde{E}, \tilde{F})$ is the trivial bundle pair $\left(\mathbb{H}^{*} \times \mathbb{C}^{N}, \mathbb{H}^{*} \times\right.$ $\left.\mathbb{R}^{N}\right)$, the lift $\tilde{\sigma}$ can be written as $\tilde{\sigma}=\left(\tilde{\sigma}_{1}, \ldots, \tilde{\sigma}_{N}\right)$, where each of the $\tilde{\sigma}_{i}: \mathbb{H}^{*} \rightarrow \mathbb{C}$ admits a Laurent series expansion

$$
\tilde{\sigma}_{i}(z)=\sum_{j=-\infty}^{\infty} a_{i, j} z^{j}
$$

with coefficients $a_{i, j} \in \mathbb{R}$. We set

$$
\tilde{\eta}_{0, i}(z):=\sum_{j=0}^{\infty} a_{i, j} z^{j}, \quad \tilde{\eta}_{1, i}(z):=\sum_{j=-\infty}^{-1} a_{i, j} z^{j}
$$

for $i=1, \ldots, N$, then $\tilde{\eta}_{0}:=\left(\tilde{\eta}_{0,1}, \ldots, \tilde{\eta}_{0, N}\right), \tilde{\eta}_{1}:=\left(\tilde{\eta}_{1,1}, \ldots, \tilde{\eta}_{1, N}\right)$, and finally

$$
\eta_{0}(z):=\left.D \pi\right|_{w(z)} \tilde{\eta}_{0}(z), \quad \eta_{1}(z):=\left.D \pi\right|_{w(z)} \tilde{\eta}_{1}(z)
$$


The $\eta_{i}$ are sections of $(E, F)$ over $\mathbb{H}^{*}$ which satisfy (1) by construction. We still have to show that they extend in the required way, which by the removal of singularities theorem requires only to show that the limits $\eta_{0}(z)$ for $z \rightarrow 0$ and $\eta_{1}(z)$ for $z \rightarrow \infty$ exist. This is clear for $\eta_{0}$, because for $z \rightarrow 0$ both $\tilde{\eta}_{0}(z)$ and $w(z)$ converge, the latter by the way $w$ was chosen. As for $\eta_{1}$, the problem is that while $\tilde{\eta}_{1}(z)$ converges to some $\tilde{\eta}_{1}(\infty), w(z)$ does not converge for $z \rightarrow \infty$. Nevertheless, we are in good shape, as the following lemma shows.

Lemma 5.7. $\left.D \pi\right|_{w(z)} \tilde{\eta}_{1}(z)$ converges to $0 \in T_{u(\infty)} R$ for $z \rightarrow \infty$.

Proof. We start by constructing a function $\theta: \mathbb{H} \rightarrow K_{\mathbb{C}}$ such that

$$
\|\theta(z) \cdot w(z)\|<C
$$

for some $C>0$ and all $z \in \mathbb{H}$. To do so, choose first an element $\lambda=\left(\lambda_{1}, \ldots, \lambda_{N}\right) \in \mathbb{K}$ such that all $\lambda_{i}$ are $>0$. This is possible, since for every $i \in[1, N]$ the vector $-v_{i}$ lies in some cone $\sigma \in \Sigma$ by the completeness of $\Sigma$ and can hence be expressed as a linear combination of the generators $v_{j_{1}}, \ldots, v_{j_{m_{i}}}$ of $\sigma$ with nonnegative coefficients. In other words, we have $v_{i}+\sum_{k=1}^{m_{i}} a_{j_{k}} v_{j_{k}}=0$ for certain $a_{j_{k}} \geq 0$. Setting $\lambda^{(i)}:=e_{i}+\sum_{k=1}^{m_{i}} a_{j_{k}} e_{j_{k}}$, where the $e_{i}$ denote the elements of the standard basis of $\mathbb{Z}^{N}$, and finally $\lambda:=\sum_{i=1}^{N} \lambda^{(i)}$ yields the required vector. Now define

$$
\theta(z):=z^{-M \lambda}=\left(z^{-M \cdot \lambda_{1}}, \ldots, z^{-M \cdot \lambda_{N}}\right),
$$

with $M:=\max _{i \in[1, N]}$ deg $w_{i}$ being the maximal degree occurring among the polynomials $w_{i}$.

By definition, we have $\pi=\pi \circ \theta(z)$ for every $z \in \mathbb{H}$, considering $\theta(z)$ as the map $\mathbb{C}^{N} \rightarrow \mathbb{C}^{N}, \xi \mapsto \theta(z) \cdot \xi$. It follows that

$$
\left.D \pi\right|_{w(z)}=\left.\left.D \pi\right|_{\theta(z) \cdot w(z)} \circ D \theta(z)\right|_{w(z)}
$$

and hence

$$
\left.D \pi\right|_{w(z)} \tilde{\eta}_{1}(z)=\left(\left.\left.D \pi\right|_{\theta(z) \cdot w(z)} \circ D \theta(z)\right|_{w(z)}\right) \tilde{\eta}_{1}(z)
$$

Now $\left.D \theta(z)\right|_{w(z)}$ converges to 0 for $z \rightarrow \infty$ by the decay property of $\tau$. Moreover, since both $\left.z \mapsto D \pi\right|_{\theta(z) \cdot w(z)}$ and $z \mapsto \tilde{\eta}_{1}(z)$ are bounded (the first because $\theta \cdot w$ only takes values in some compact subset of $\mathbb{C}^{N}$ by the decay of $\theta$, and the second because it converges), we obtain $D \pi_{w(z)} \tilde{\eta}(z) \rightarrow 0$. 
This completes the proof of the claim and hence of the disc part of Theorem 5.1.

\subsection{Regularity for spheres}

The proof of the sphere part of Theorem 5.1 follows the same pattern as that of the disc part. Let $v: \mathbb{C} P^{1} \rightarrow X$ be an arbitrary holomorphic sphere. As in the disc case, proving regularity of $J_{0}$ at $v$ amounts to showing that the Dolbeault operator $\bar{\partial}: C^{\infty}\left(v^{*} T X\right) \rightarrow$ $\Omega^{0,1}\left(v^{*} T X\right)$ has coker $\bar{\partial}=0$. By Proposition 5.2, which also holds for closed Riemann surfaces, this translates into $H^{1}\left(\mathbb{C} P^{1} ; \mathcal{O}\left(v^{*} T X\right)\right)=0$, where $\mathcal{O}\left(v^{*} T X\right)$ denotes the sheaf of holomorphic sections of $v^{*} T X$.

By arguments similar to the ones in the proof of Proposition 4.2, the restriction of $v$ to any subset $\mathbb{C} P^{1} \backslash\{\mathrm{pt}\}$ admits a lift $w=\left(w_{1}, \ldots, w_{N}\right)$ to $\mathbb{C}^{N}$ with polynomial entries $w_{i}$. One obtains the exact sequence of sheaves

$$
0 \rightarrow \mathcal{O}\left(w^{*}\left(\operatorname{TOrb}_{K_{\mathbb{C}}}\right)\right) \rightarrow \mathcal{O}\left(w^{*} \mathbb{C}^{N}\right) \rightarrow \mathcal{O}\left(v^{*} T X\right) \rightarrow 0
$$

and then deduces from the resulting long exact sequence in cohomology that the cover $\mathfrak{V}$ of $\mathbb{C} P^{1}$ given by $V_{0}=\mathbb{C} P^{1} \backslash\{\infty\}$ and $V_{1}=\mathbb{C} P^{1} \backslash\{0\}$ is acyclic for $\mathcal{O}\left(v^{*} T X\right)$. The vanishing of $H^{1}\left(\mathbb{C} P^{1} ; \mathcal{O}\left(v^{*} T X\right)\right) \cong \check{H}{ }^{1}\left(\mathfrak{V} ; \mathcal{O}\left(v^{*} T X\right)\right.$ is then shown by the same Čech type argument as in the previous section: given a holomorphic section $\sigma$ of $v^{*} T X$ over $V_{0} \cap V_{1}$, lift it to a holomorphic section of the trivial bundle, decompose it using its Laurent expansion as $\sigma=\eta_{0}+\eta_{1}$ such that $\eta_{0}$ extends over 0 and $\eta_{1}$ extends over $\infty$, and then project back to $v^{*} T X$.

\section{The Involution on the Moduli Space of Real Discs}

Denote by $H_{2}^{D}(X, R)$ the image of the Hurewicz homomorphism $\pi_{2}(X, R) \rightarrow H_{2}(X ; \mathbb{Z})$ and by $\sim$ the equivalence relation on $H_{2}^{D}(X, R)$ given by identifying classes of the same Maslov number:

$$
\lambda \sim \lambda^{\prime} \quad: \Longleftrightarrow \mu(\lambda)=\mu\left(\lambda^{\prime}\right)
$$

Let $\tilde{\mathcal{M}}(\lambda)$ be the moduli space of parametrized holomorphic discs $u:\left(D^{2}, \partial D^{2}\right) \rightarrow(X, R)$ representing the class $\lambda \in H_{2}^{D}(X, R) / \sim$. Then define the corresponding moduli space of unparametrized discs with two marked boundary points by

$$
\mathcal{M}_{2}(\lambda):=\tilde{\mathcal{M}}(\lambda) / \operatorname{Aut}_{ \pm 1}\left(D^{2}\right)
$$


where $\operatorname{Aut}_{ \pm}\left(D^{2}\right)$ denotes the subgroup of $\operatorname{Aut}\left(D^{2}\right)$ consisting of those automorphism which fix $\pm 1 \in \partial D^{2}$. Furthermore, set

$$
\mathcal{M}_{2}:=\bigsqcup_{\lambda} \mathcal{M}_{2}(\lambda)
$$

We commonly denote elements of $\mathcal{M}_{2}$ by the same name as their representatives, which should cause no confusion. Note that it makes sense to evaluate $u \in \mathcal{M}_{2}$ at the points \pm 1 .

\subsection{The involution $\tau_{*}$ and its fixed points}

In this section we will study the involution

$$
\begin{aligned}
\tau_{*}: \mathcal{M}_{2} & \rightarrow \mathcal{M}_{2}, \\
u & \mapsto \tau \circ u \circ c,
\end{aligned}
$$

where $c: D^{2} \rightarrow D^{2}$ denotes complex conjugation. Its fixed point set was characterized by Fukaya et al. [17] (which is part of the big FOOO preprint, but is not contained in the book version) in terms of another map

$$
\mathfrak{D}: \mathcal{M}_{2} \rightarrow \mathcal{M}_{2}
$$

We briefly describe its definition. Denote by $D_{ \pm}^{2}:=\left\{z \in D^{2} \mid \pm \operatorname{Im} z \geq 0\right\}$ the upper respectively lower half-disc, and let $\rho_{ \pm}: D_{ \pm}^{2} \rightarrow D^{2}$ be conformal isomorphisms satisfying $\rho_{-}(z)=\overline{\rho_{+}(\bar{z})}$. (In the upper half-plane model, in which $D_{ \pm}^{2}$ correspond to $\mathbb{H}_{ \pm}=\{z \in \mathbb{H} \mid$ $\pm \operatorname{Re} z \geq 0\}$, such maps are given by $\mathbb{H}_{ \pm} \rightarrow \mathbb{H}, z \mapsto \pm z^{2}$.) Given a real disc $u \in \tilde{\mathcal{M}}_{2}(\lambda)$, define a new real disc $u^{\prime} \in \tilde{\mathcal{M}}_{2}\left(\lambda-\tau_{*} \lambda\right)=\tilde{\mathcal{M}}_{2}(2 \lambda)$ by

$$
\check{u}:= \begin{cases}u\left(\rho_{-}(z)\right), & z \in D_{-}^{2}, \\ \tau\left(u\left(\overline{\left.\rho_{+}(z)\right)}\right),\right. & z \in D_{+}^{2} .\end{cases}
$$

It is shown in [17] (Lemma 40.4) that $\mathfrak{D}$ descends to a map $\mathfrak{D}: \mathcal{M}_{2} \rightarrow \mathcal{M}_{2}$.

The fixed point set of $\tau_{*}$ is simply the image of $\mathfrak{D}$ :

Lemma 6.1 (see [17, Lemma 40.6]). Given any $u \in \mathcal{M}_{2}$ which is fixed by $\tau_{*}$, there exists some $u^{\prime} \in \mathcal{M}_{2}$ such that $\mathfrak{D} u^{\prime}=u$. 


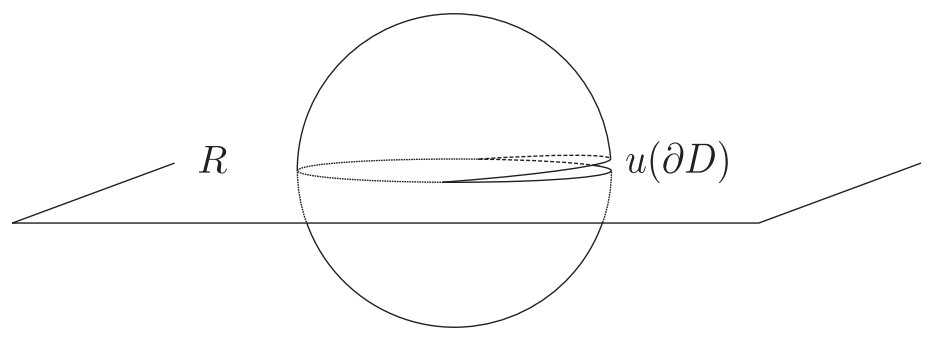

Fig. 1. A Pac-Man disc.

The fixed points can be pictured as illustrated in Figure 1 (the mouth of the PacMan, which corresponds to $\partial D^{2}$, should really be entirely closed).

\subsection{A closer look at the fixed points}

The restrictions of a real rational curve $v: \mathbb{C} P^{1} \rightarrow X$ to the upper respectively lower hemispheres yield two real discs

$$
v_{ \pm}:\left(D^{2}, \partial D^{2}\right) \rightarrow(X, R),
$$

which we call the upper and lower halves of $v$. In general, the $v_{ \pm}$are not distinct as elements of $\mathcal{M}_{2}$.

Let now $u \in \mathcal{M}_{2}$ be a fixed point of $\tau_{*}$, and let $u^{\prime} \in \mathcal{M}_{2}$ be such that $\mathfrak{D} u^{\prime}=u$. Since every real disc can be obtained as one half of its double, we have in particular $u^{\prime}=v_{ \pm}$ for a real rational curve $v$ and can write

$$
u=\mathfrak{D} v_{ \pm}
$$

The next proposition formulates a dichotomy based on whether $v$ is simple or not. Recall that simplicity for a holomorphic sphere $v: \mathbb{C} P^{1} \rightarrow X$ means that it is not multiply covered, that is, it cannot be written as $v=v^{\prime} \circ \phi$, where $v^{\prime}: \mathbb{C} P^{1} \rightarrow X$ is another holomorphic sphere and $\phi: \mathbb{C} P^{1} \rightarrow \mathbb{C} P^{1}$ is a holomorphic branched cover of degree $>1$.

Proposition 6.2. Let $v: \mathbb{C} P^{1} \rightarrow X$ be a real rational curve.

(i) If $v$ is simple, then $\mathfrak{D} v_{+}$and $\mathfrak{D} v_{-}$are distinct as elements of $\mathcal{M}_{2}$.

(ii) If $v$ is nonsimple, then there exist real discs $u_{ \pm}$satisfying

- $\mu\left(u_{ \pm}\right)<\mu\left(\mathfrak{D} v_{ \pm}\right)$;

- $\left(u_{ \pm}\right)\left(D^{2}\right)=\left(\mathfrak{D} v_{ \pm}\right)\left(D^{2}\right)$. 
Proof. (i) Suppose that $v$ is simple. In the usual identification $\mathbb{C} P^{1} \backslash\{\infty\} \cong \mathbb{C}$, the discs $v_{ \pm}$are simply the restrictions of $v$ to the upper and lower half-planes, and the $\mathfrak{D} v_{ \pm}=: \check{v}_{ \pm}$ are given by $z \mapsto v\left( \pm z^{2}\right)$ for $z \in \mathbb{H} \subset D^{2}$. The images of $\partial D^{2}$ under $\check{v}_{ \pm}$are hence

$$
\check{v}_{+}\left(\partial D^{2}\right)=v\left(\overline{\mathbb{R}}_{+}\right) \quad \text { and } \quad \check{v}_{-}\left(\partial D^{2}\right)=v\left(\overline{\mathbb{R}}_{-}\right)
$$

where $\overline{\mathbb{R}}_{ \pm}=\mathbb{R}_{ \pm} \cup\{\infty\}$ denote the extended positive and negative half-lines.

Suppose that $\check{v}_{+}$and $\check{v}_{-}$define the same element of $\mathcal{M}_{2}$. Then it follows, in particular, that $\check{v}_{+}\left(\partial D^{2}\right)=\check{v}_{-}\left(\partial D^{2}\right)$, and hence $v\left(\overline{\mathbb{R}}_{+}\right)=v\left(\overline{\mathbb{R}}_{-}\right)$. So the set of noninjective points of $v$ contains at least all of $\mathbb{R} P^{1}$, contradicting the fact that this set is at most countable for any simple curve, see [22, Proposition 2.5.1].

(ii) If $v$ is nonsimple, we can by definition write $v=v^{\prime} \circ \phi$ with $v^{\prime}: \mathbb{C} P^{1} \rightarrow X$ another holomorphic sphere and a $\phi: \mathbb{C} P^{1} \rightarrow \mathbb{C} P^{1}$ a branched cover of degree $>1$.

Claim. Such $v^{\prime}$ can be chosen among real rational curves.

Observe that the statement of part (ii) of the proposition follows from the claim, because we can then define the required discs $u_{ \pm}$by

$$
u_{ \pm}:=v_{ \pm}^{\prime}
$$

that is, as the upper respectively lower halves of $v^{\prime}$. These clearly have the required properties.

Proof of the Claim. We first construct a Riemann surface $(\Sigma, j)$ from the image of $v$, together with a $j$-anti-holomorphic involution $v: \Sigma \rightarrow \Sigma$ and a holomorphic map $f: \Sigma \rightarrow X$ satisfying $f=\tau \circ f \circ v$. Then we argue that $\Sigma$ is biholomorphic to $\mathbb{C} P^{1}$ by a biholomorphism which is intertwines $v$ and complex conjugation.

As for the construction of $\Sigma$, we follow closely the exposition in the proof of McDuff and Salamon [22, Proposition 2.5.1] (and refer there for more details), where essentially the same appears. Denote by $C \subset \operatorname{Im}(v)$ the set of critical values of $v$, and let $B$ be the subset of $\operatorname{Im}(v) \backslash C$ consisting of all those points where distinct branches of $v$ meet. That is, a point $x \in \operatorname{Im}(v)$ is in $B$ iff there exist distinct $z_{0}, z_{1} \in \mathbb{C} P^{1} \backslash v^{-1}(C)$ such that $v\left(z_{1}\right)=v\left(z_{2}\right)$ and such that for all sufficiently small neighborhoods $U_{i}$ of $z_{i}$ we have $v\left(U_{0}\right) \neq v\left(U_{1}\right)$. Since $B$ is a discrete subset of $\operatorname{Im}(v)$, it follows that

$$
\Sigma^{\prime}:=\operatorname{Im}(v) \backslash(B \cup C)
$$


is an embedded submanifold. We denote the embedding by $\iota: \Sigma^{\prime} \rightarrow X$. $\Sigma^{\prime}$ carries a unique complex structure $j^{\prime}$ such that $\iota$ is holomorphic. Furthermore, $\Sigma^{\prime}$ has two types of ends, namely those corresponding to the points in $B$ (a finite number for each $x \in B$, one for each branch of $v$ through $x$ ), and those corresponding to the points in $C$. By adding one point to $\Sigma^{\prime}$ for each end and extending the complex structure, we obtain a closed Riemann surface $(\Sigma, j)$, and the embedding $\iota$ extends to a holomorphic map $f: \Sigma \rightarrow X$.

The Riemann surface $\left(\Sigma^{\prime}, j^{\prime}\right)$ carries an anti-holomorphic involution $v$ induced by $\tau$, that is, given by $v=\iota^{-1} \circ \tau \circ \iota$, which extends to an anti-holomorphic involution of $(\Sigma, j)$. Furthermore, $f$ satisfies $f=\tau \circ f \circ \sigma$.

By construction, $\Sigma$ admits a branched cover $\mathbb{C} P^{1} \rightarrow \Sigma$ and must hence itself be biholomorphic to $\mathbb{C} P^{1}$. We argue that there exists a biholomorphism $\psi: \mathbb{C} P^{1} \rightarrow \Sigma$ intertwining complex conjugation and $v$. Indeed, this follows from the fact that complex conjugation is the only anti-holomorphic involution on $\mathbb{C} P^{1}$ (up to conjugation with an automorphism) having a nondiscrete set of fixed points, which is also the case for $v$ by construction.

Finally, setting

$$
v^{\prime}:=f \circ \psi: \mathbb{C} P^{1} \rightarrow X
$$

produces the required real rational curve, which ends the proof of the claim and hence of the proposition.

\section{The Pearly Moduli Spaces}

In this section we recall from [6, 7] the definitions of the moduli spaces appearing in the construction of the quantum homology rings $\mathrm{OH}\left(R ; \Lambda_{R}\right)$ and $\mathrm{OH}\left(X ; \Lambda_{X}\right)$, and show that the necessary auxiliary structures may be chosen among $\tau$-(anti)-invariant ones, which is essential for the proof of Theorem A to work.

\subsection{Definitions}

Fix a triple $\mathcal{D}=(f, g, J)$, where $f: X \rightarrow \mathbb{R}$ is a Morse function, $g$ a Riemannian metric on $X$ and $J$ an almost complex structure on $X$. Denote for now by $f$ and $g$ also the restrictions to $R$, and by $\Phi_{t}$ the negative gradient flow of $f$. We consider three different types of moduli spaces, which appear in the definitions of the Lagrangian quantum differential and the quantum products on $\mathrm{QH}\left(R ; \Lambda_{R}\right)$ respectively $\mathrm{OH}\left(X ; \Lambda_{X}\right)$. (A graphical depiction of the elements of these moduli space is provided in Figure 2.) 

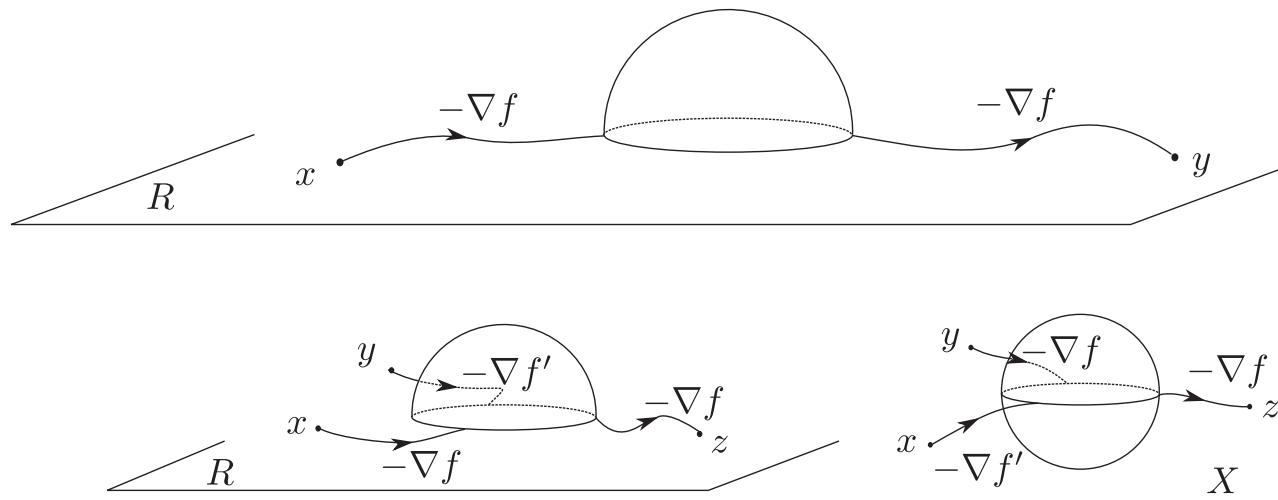

Fig. 2. Elements of the pearly moduli spaces.

\subsubsection{Pearly trajectories}

Given points $x, y \in R$ (not necessarily critical) and a class $\lambda \in H_{2}^{D}(X, R) / \sim$ (recall that $\sim$ identifies classes of the same Maslov number), denote by $\mathcal{P}(x, y, \lambda ; \mathcal{D})$ the set of all tuples $\left(u_{1}, \ldots, u_{\ell}\right) \in \mathcal{M}_{2}^{\times \ell}$ such that

1. All $u_{i}$ are nonconstant.

2. $\Phi_{t_{0}}\left(u_{1}(-1)\right)=x$ for some $-\infty \leq t_{0}<0$.

3. $\Phi_{t_{i}}\left(u_{i}(+1)\right)=u_{i+1}(-1)$ for some $0<t_{i}<\infty, i \in[1, \ell-1]$.

4. $\Phi_{t_{\ell}}\left(u_{\ell}(+1)\right)=y$ for some $0<t_{\ell} \leq \infty$.

5. $\left[u_{1}\right]+\cdots+\left[u_{\ell}\right]=\lambda$.

Here $\ell$ is not supposed to be fixed. If $x$ and $y$ are critical points of $f$, the virtual dimension of this space is

$$
\operatorname{dim}_{\text {virt }} \mathcal{P}(x, y, \lambda ; \mathcal{D})=|x|-|y|+\mu(\lambda)-1,
$$

where $|\cdot|$ denotes the Morse index of $f: R \rightarrow \mathbb{R}$.

\subsubsection{Discy Y-pearls}

Choose a second Morse function $f^{\prime}: X \rightarrow \mathbb{R}$ such that all the (un)stable submanifolds of $f$ and $f^{\prime}$ intersect transversely. Let $x, z \in \operatorname{Crit} f, y \in \operatorname{Crit} f^{\prime}, \lambda \in H_{2}^{D}(M, L) / \sim$. Then define $\mathcal{Y}^{D}\left(x, y, z, \lambda ; \mathcal{D}, \mathcal{D}^{\prime}\right)$ to be the set of all tuples $\left(\mathbf{u}, \mathbf{u}^{\prime}, \mathbf{u}^{\prime \prime}, u_{\mathrm{c}}\right)$ where

1. $u_{\mathrm{c}}:(D, \partial D) \rightarrow(X, R)$ is a real disc, possibly constant.

2. $\mathbf{u} \in \mathcal{P}\left(x, u_{\mathrm{c}}\left(\mathrm{e}^{-2 \pi \mathrm{i} / 3}\right), \eta ; f, g, J\right)$. 
3. $\mathbf{u}^{\prime} \in \mathcal{P}\left(y, u_{\mathrm{c}}\left(\mathrm{e}^{2 \pi \mathrm{i} / 3}\right), \eta^{\prime} ; f^{\prime}, g, J\right)$.

4. $\mathbf{u}^{\prime \prime} \in \mathcal{P}\left(u_{\mathrm{c}}(1), z, \eta^{\prime \prime} ; f, g, J\right)$.

5. $\eta, \eta^{\prime}, \eta^{\prime \prime} \in H_{2}^{D}(x, R) / \sim$ satisfy $\eta+\eta^{\prime}+\eta^{\prime \prime}+\left[u_{\mathrm{c}}\right]=\lambda$.

We will later refer to $u_{c}$ as the central disc. The virtual dimension in this case is

$$
\operatorname{dim}_{\text {virt }} \mathcal{Y}^{D}\left(x, y, z, \lambda ; \mathcal{D}, \mathcal{D}^{\prime}\right)=|x|+|y|-|z|+\mu(\lambda)-n
$$

with $|\cdot|$ denoting again the Morse index of $f: R \rightarrow \mathbb{R}$.

\subsubsection{Spherical Y-pearls}

Denote by $H_{2}^{S}(X)$ the image of the Hurewicz homomorphism $\pi_{2}(X) \rightarrow H_{2}(X ; \mathbb{Z})$ and by $\sim$ the equivalence relation given by identifying classes of the same Chern number. Again, let $f^{\prime}: X \rightarrow \mathbb{R}$ be a second Morse function and denote its negative gradient flow by $\Phi_{t}^{\prime}$. Given some $x, z \in$ Crit $f$ and $y \in \operatorname{Crit} f^{\prime}$ and $\lambda \in H_{2}^{S}(X) / \sim$, let $\mathcal{Y}^{S}\left(x, y, z, \lambda ; \mathcal{D}, \mathcal{D}^{\prime}\right)$ be the space of all holomorphic spheres $v: \mathbb{C} P^{1} \rightarrow X$ such that

1. $\Phi_{-\infty}\left(v\left(\mathrm{e}^{-2 \pi \mathrm{i} / 3}\right)\right)=X$.

2. $\Phi_{-\infty}^{\prime}\left(v\left(\mathrm{e}^{2 \pi \mathrm{i} / 3}\right)\right)=y$.

3. $\Phi_{+\infty}(v(1))=z$.

4. $[v]=\lambda$.

The virtual dimension of this space is

$$
\operatorname{dim}_{\text {virt }} \mathcal{Y}^{S}\left(x, y, z, \lambda ; \mathcal{D}, \mathcal{D}^{\prime}\right)=|x|+|y|-|z|+2 c_{1}(\lambda)-2 n
$$

where now $|\cdot|$ denotes the Morse index of $f, f^{\prime}: X \rightarrow \mathbb{R}$.

\subsection{Regularity}

This subsection serves to justify working with the following data: the standard $\tau$-antiinvariant complex structure $J_{0}$ on $X$ and a generic pair $\left(f_{\xi}, g\right)$, where $f_{\xi}: X \rightarrow \mathbb{R}$ is defined by $f_{\xi}(x)=\langle\mu, \xi\rangle$ with some $\xi \in \mathfrak{q}$ as before, and $g \in \mathcal{G}_{\tau}$ is a $\tau$-invariant Riemannian metric on $X$. More precisely, we prove the following proposition.

Proof of Proposition 7.1. There exist second category subsets of $\mathfrak{q} \times \mathcal{G}_{\tau}$ resp. $\mathfrak{q} \times \mathfrak{q} \times \mathcal{G}_{\tau}$ such that for all $(\xi, g)$ resp. $\left(\xi, \xi^{\prime}, g\right)$ in these subsets all moduli spaces $\mathcal{P}(x, y, \lambda ; \mathcal{D})$ 
resp. $\mathcal{Y}^{D}\left(x, y, z, \lambda ; \mathcal{D}, \mathcal{D}^{\prime}\right)$ and $\mathcal{Y}^{S}\left(x, y, z, \lambda ; \mathcal{D}, \mathcal{D}^{\prime}\right)$ defined using data triples $\mathcal{D}=\left(f_{\xi}, g, J_{0}\right)$ resp. pairs of data triples $\mathcal{D}=\left(f_{\xi}, g, J_{0}\right)$ and $\mathcal{D}^{\prime}=\left(\tilde{f}_{\xi^{\prime}}, g, J_{0}\right)$ are smooth manifolds of the respective virtual dimension.

Remark. As in the case of the Morse intersection product (see the end of Section 3.2), we use as the Morse function in the second triple used for the definition of the quantum product a slightly perturbed version $\tilde{f}_{\xi^{\prime}}$ of $f_{\xi^{\prime}}$, such as to ensure that the critical sets of $f_{\xi}$ and $\tilde{f}_{\xi^{\prime}}$ are disjoint.

Proof. The proof uses only standard methods. We give an outline for the case $\mathcal{P}(x, y, z, \lambda ; \mathcal{D})$, the other cases being similar. Let $\lambda=\left(\lambda_{1}, \ldots, \lambda_{\ell}\right)$ be a vector of nonzero homology classes $\lambda_{i} \in H_{2}^{D}(X, R)$ and set

$$
\mathcal{M}_{2}(\lambda):=\mathcal{M}_{2}\left(\lambda_{1}\right) \times \cdots \times \mathcal{M}_{2}\left(\lambda_{\ell}\right)
$$

which is a finite dimensional manifold. Then set

$$
\mathcal{W}_{\ell}^{1,2}:=W^{1,2}((-\infty, 0], R) \times\left(W^{1,2}([0,1], R)\right)^{\times(\ell-1)} \times W^{1,2}([0, \infty), R)
$$

where $W^{1,2}(I, R)$ denotes the space of paths $\gamma: I \rightarrow R$ of Sobolev class $W^{1,2}$. It is well known that $W^{1,2}(I, R)$ is a smooth Banach manifold, and hence $\mathcal{W}_{\ell}^{1,2}$ is so, too. Consider now the map

$$
\begin{gathered}
E V: \mathcal{M}_{2}(\lambda) \times \mathcal{W}_{\ell}^{1,2} \rightarrow \mathcal{M}_{2}(\lambda) \times R^{2 \ell+2}, \\
(\mathbf{u}, \boldsymbol{\gamma}) \mapsto\left(\mathbf{u},\left(\gamma_{0}(-\infty), \gamma_{0}(0), \gamma_{1}(0), \ldots, \gamma_{\ell}(\infty)\right)\right)
\end{gathered}
$$

This map is a submersion, so that the preimage under $E V$ of every submanifold of $\mathcal{M}_{2}(\lambda) \times R^{2 \ell+2}$ is a submanifold. In particular, the set

$$
\mathcal{X}:=E V^{-1}\left\{\left(\mathbf{u},\left(x, u_{1}(-1), \ldots, u_{\ell}(+1), y\right)\right) \in \mathcal{M}_{2}(\lambda) \times R^{2 \ell+2} \mid u \in \mathcal{M}_{2}(\lambda)\right\}
$$

is a submanifold of $\mathcal{M}_{2}(\lambda) \times \mathcal{W}_{\ell}^{1,2}$, because the set on the right-hand side is clearly a submanifold of $\mathcal{M}_{2}(\lambda) \times R^{2 \ell+2}$.

Fix now some $k>0$ and let $\mathcal{G}_{\tau}^{k}$ be the set of $\tau$-invariant metrics of class $C^{k}$, which is a Banach manifold. Consider then the Banach bundle $\mathcal{E} \rightarrow \mathcal{X} \times \mathfrak{q} \times \mathcal{G}_{\tau}^{k}$ whose fibre at 
$(\mathbf{u}, \boldsymbol{\gamma}, \xi, g)$ is

$$
\mathcal{E}_{(\mathbf{u}, \boldsymbol{\gamma}, \xi, g)}=L^{2}\left(\boldsymbol{\gamma}^{*} T R\right)
$$

Define a section $\psi$ of this bundle by

$$
\psi(\mathbf{u}, \boldsymbol{\gamma}, \xi, g):=\boldsymbol{\gamma}^{\prime}-V_{\xi} \circ \boldsymbol{\gamma}:=\left(\gamma_{0}^{\prime}-V_{\xi} \circ \gamma_{0}, \ldots, \gamma_{\ell}^{\prime}-V_{\xi} \circ \gamma_{\ell}\right)
$$

where $V_{\xi}$ denotes the negative gradient vector field of $f_{\xi}$ with respect to $g$. Observe that for given $(\xi, g)$, there is a natural 1-1 correspondence

$$
\{(\mathbf{u}, \boldsymbol{\gamma}) \in \mathcal{X} \mid \psi(\mathbf{u}, \boldsymbol{\gamma}, \xi, g)=0\} \cong \mathcal{P}\left(x, y, \lambda ;\left(f_{\xi}, g, J_{0}\right)\right)
$$

where the set on the right-hand side denotes the space of pearly trajectories whose discs represent the vector $\lambda$.

Claim. For every $(\mathbf{u}, \boldsymbol{\gamma}, \xi, g) \in \psi^{-1}(0)$, the following assertions hold true:

(i) The vertical differential $D^{v} \psi: T_{(\mathbf{u}, \boldsymbol{\gamma}, \xi, g)}\left(\mathcal{X} \times \mathfrak{q} \times T_{g} \mathcal{G}_{\tau}^{k}\right) \rightarrow \mathcal{E}_{(\mathbf{u}, \boldsymbol{\gamma}, \xi, g)}$ is onto.

(ii) The restricted vertical differential $D_{1}^{v} \psi: T_{(\mathbf{u}, \boldsymbol{\gamma})} \mathcal{X} \rightarrow \mathcal{E}_{(\mathbf{u}, \boldsymbol{\gamma}, \xi, g)}$ is a Fredholm operator of index $|x|-|y|+\mu(\lambda)-1$.

Once this is proved, the statement of the proposition will follow by first applying a standard theorem (see, e.g., [20]), which says that under these conditions the set of $(\xi, g) \in \mathfrak{q} \times \mathcal{G}_{\tau}^{k}$ for which $\{(\mathbf{u}, \boldsymbol{\gamma}) \mid \psi(\mathbf{u}, \boldsymbol{\gamma}, \xi, g)=0\} \subset \mathcal{M}_{2}(\lambda) \times \mathcal{W}_{\ell}^{1,2}$ is a smooth submanifold of the right dimension is of second category, and then using a procedure due to Taubes to get from $C^{k}$ to $C^{\infty}$ (see the proof of Theorem 3.1.5 (ii) in [22]).

We omit the proof of assertion (ii), which is standard. As for (i), note that $D^{v} \psi$ evaluated on a tangent vector of the form $(0,0,0, \dot{g})$ with $\dot{g} \in T_{g} \mathcal{G}_{\tau}^{k}$ yields

$$
D^{v} \psi(0,0,0, \dot{g})=-\dot{V}_{\xi} \circ \gamma
$$

where $\dot{V}_{\xi}=-g^{-1} \dot{g}\left(V_{\xi}\right)$ is the variation of the gradient vector field induced by $\dot{g}$ (here we view $g, \dot{g}$ as sections of $\left.\operatorname{Hom}\left(T X, T^{*} X\right)\right)$. Let $W \in L^{2}\left(\gamma^{*} T R\right)$ be any element orthogonal to the range of $D_{1}^{v} \psi$ (which is a closed subspace of $\mathcal{E}_{(\mathbf{u}, \gamma, \xi, g)}$, since $D_{1}^{v} \psi$ is Fredholm). Then 
in particular

$$
\int_{\gamma} g\left(\dot{V}_{\xi}, W\right) \mathrm{d} s=0
$$

for all $\dot{g} \in T_{g} \mathcal{G}_{\tau}^{k}$. Now given any point $p \in \operatorname{im} \gamma \in R$, one can easily find some $\dot{g}(p) \in$ $\operatorname{Hom}\left(T_{p} R, T_{p}^{*} R\right)$ such that $\dot{V}_{f_{\xi}}(p)=W(p)$ for the corresponding $\dot{V}_{\xi}$. Here we assume $W$ to be continuous, which is legal by density of continuous sections in $L^{2}$. Extending $\dot{g}(p)$ by using a $\tau$-invariant cutoff function supported near $p$ yields an element $\dot{g} \in T_{g} \mathcal{G}_{\tau}^{k}$, and the fact that it pairs to zero with $W$ allows to conclude that $W(p)=0$. This argument works because $\gamma$ consists of pieces of gradient flow lines, which cannot intersect each other. So $W \equiv 0$, and hence $D_{1}^{v} \psi$ is surjective.

\subsection{Compactness}

To be able to count the elements of the zero-dimensional pearly moduli spaces, we need the following proposition.

Proposition 7.1. Let $\mathcal{D}, \mathcal{D}^{\prime}$ be generic data triples in the sense of Proposition 7.1. Then all moduli spaces $\mathcal{P}(x, y, \lambda ; \mathcal{D}), \mathcal{Y}^{D}\left(x, y, z, \lambda ; \mathcal{D}, \mathcal{D}^{\prime}\right)$, and $\mathcal{Y}^{S}\left(x, y, z, \lambda ; \mathcal{D}, \mathcal{D}^{\prime}\right)$ of dimension zero are compact, that is, finite sets.

The proof of this proposition is the point where the assumptions of monotonicity and $C_{X} \geq 2$ are needed. Given that these are satisfied, it requires no arguments which are special to our situation, but follows directly from Biran-Cornea's general theory. The idea for proving compactness in dimension zero is that if there were some sequence without a converging subsequence, then a subsequence would have to Gromov-converge to a pearly configuration in some other space, for example, by bubbling off of a sphere. From this one could extract a configuration in a pearly moduli space of negative virtual dimension (in our example by forgetting the sphere), contradicting the fact that such a space must be empty by Proposition 7.1. For a precise version of this argument, see [6].

\subsection{A toy example}

As an illustration we work out the pearly moduli spaces relevant for the quantum products of $(X, R)=\left(\mathbb{C} P^{1}, \mathbb{R} P^{1}\right)$. Identifying $\mathbb{C} P^{1}$ with $S^{2} \subset \mathbb{R}^{3}$ in a standard way, the Hamiltonian $S^{1}$-action is given by rotations around the $z$-axis and $\mathbb{R} P^{1}$ is identified with some equator. Given some $\xi$ in the Lie algebra of $S^{1}$, the corresponding $f_{\xi}$ is a multiple of the 


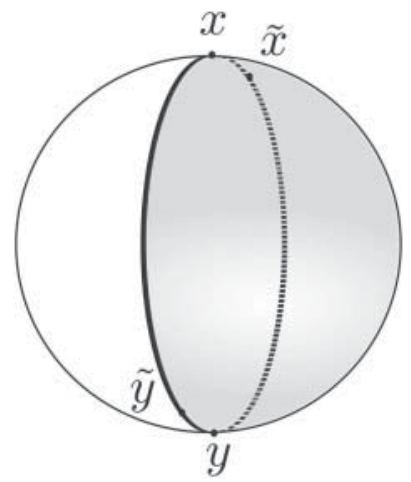

Fig. 3. $(X, R)=\left(\mathbb{C} P^{1}, \mathbb{R} P^{1}\right)$.

height function, whose critical points we label $x$ and $y$. We also choose a small perturbation $\tilde{f}_{\xi}$ of $f_{\xi}$ whose critical points $\tilde{X}, \tilde{y}$ are different from $x, y$. These Morse functions together with the round metric and the standard complex structure constitute regular data tuples with which we can compute the nonclassical part of the quantum product on $\mathrm{OH}\left(R ; \Lambda_{R}\right)$ and $\mathrm{OH}\left(X ; \Lambda_{X}\right)$. For degree reasons, the only zero-dimensional moduli spaces of Y-pearls involving nonconstant discs are $\mathcal{Y}^{D}\left(y, \tilde{Y}, x, \lambda_{ \pm}\right)$and $\mathcal{Y}^{S}(y, \tilde{Y}, x, \lambda)$, with $\lambda_{ \pm} \in H_{2}^{D}(X, R)$ denoting the two $\tau$-invariant classes of Maslov index 2 and $\lambda \in H_{2}(X)$ the fundamental class. Assuming that the critical points are positioned as in Figure 3 and that the shaded disc represents $\lambda_{+}$, we conclude that $\mathcal{Y}^{D}\left(y, \tilde{Y}, x, \lambda_{+}\right)$contains precisely one element, which "is" the shaded disc, thought of as a holomorphic disc connected to $x, y, \tilde{y}$ by gradient flow lines of length zero. Conversely, $\mathcal{Y}^{D}\left(y, \tilde{y}, x, \lambda_{-}\right)$is empty, because the boundary of the nonshaded disc, which represents $\lambda_{-}$, passes through $x, y, \tilde{Y}$ in the wrong order. $\mathcal{Y}^{S}(y, \tilde{Y}, x, \lambda)$ also contains precisely one element, namely the holomorphic sphere represented by $\operatorname{id}_{\mathbb{C} P^{1}}$. From this it is easy to conclude that the the ring structures of $\mathrm{OH}\left(R ; \Lambda_{R}\right)$ and $\mathrm{OH}\left(X ; \Lambda_{X}\right)$ are as described in the Section 1 (see Section 8 for a reminder about the definition of the quantum products.)

\section{Proof of Theorem A}

\subsection{Proof of Theorem A(i)}

We briefly recall the definition of the quantum homology $\mathrm{OH}\left(R ; \Lambda_{R}\right)$, referring to [6, 7] for details. Given a data triple $\mathcal{D}=(f, g, J)$, define the graded vector space

$$
\mathcal{C}\left(\mathcal{D} ; \Lambda_{R}\right)=\mathbb{Z}_{2}\langle\text { Crit } f\rangle \otimes \Lambda_{R}
$$


whose grading is induced by the Morse index of $f$ and the grading of $\Lambda_{R}$. Consider the $\Lambda_{R}$-linear homomorphism $d: \mathcal{C}\left(\mathcal{D} ; \Lambda_{R}\right) \rightarrow \mathcal{C}\left(\mathcal{D} ; \Lambda_{R}\right)$ given by

$$
\mathrm{d} x=\sum_{y, \lambda}\left(\#_{\mathbb{Z}_{2}} \mathcal{P}(x, y, \lambda)\right) y t^{\mu(\lambda) / N_{R}},
$$

for $x \in$ Crit $f$, where the sum runs over all $y \in$ Crit $f$ and all $\lambda \in H_{2}^{D}(X, R) / \sim$ such that $|x|-|y|-\mu(\lambda)-1=0$. Assuming a generic choice of $\mathcal{D}$, the count makes sense, because then $\mathcal{P}(x, y, \lambda)$ is a compact zero-dimensional manifold, that is, a finite set of points. Moreover, $d$ is a differential, that is, $d^{2}=0$. The quantum homology of $R$ is then defined as

$$
\mathrm{OH}\left(R ; \Lambda_{R}\right):=\mathrm{OH}\left(\mathcal{D} ; \Lambda_{R}\right):=H\left(\mathcal{C}\left(\mathcal{D} ; \Lambda_{R}\right), d\right)
$$

and is independent of the data up to isomorphism, in the sense that given another data triple $\mathcal{D}^{\prime}$ there exists a chain map $\mathcal{C}\left(\mathcal{D} ; \Lambda_{R}\right) \rightarrow \mathcal{C}\left(\mathcal{D}^{\prime} ; \Lambda_{R}\right)$ inducing a canonical isomorphism

$$
\Psi_{\mathcal{D}^{\prime}, \mathcal{D}}: \mathrm{OH}\left(\mathcal{D} ; \Lambda_{R}\right) \rightarrow \mathrm{OH}\left(\mathcal{D}^{\prime} ; \Lambda_{R}\right)
$$

We now specialize to the case that $\left(f_{\xi}, g_{0}, J_{0}\right)$ is a triple as in Section 7 , with $f_{\xi} \in \mathcal{F}_{0}$ chosen such as to make all the corresponding pearly moduli spaces manifolds of the expected dimension. We know that we compute the right quantum invariants when working with such data, because we can relate the corresponding moduli spaces to those for arbitrary generic data by a compact cobordism. This follows from the regularity results in Section 7 and the general theory of Biran-Cornea.

Claim. For this choice of data $\mathcal{D}=\left(f_{\xi}, g_{0}, J_{0}\right)$, the corresponding homomorphism

$$
d: \mathcal{C}\left(\mathcal{D} ; \Lambda_{R}\right) \rightarrow \mathcal{C}\left(\mathcal{D} ; \Lambda_{R}\right)
$$

vanishes.

The wideness statement of Theorem A follows immediately from the claim: The vanishing of $d$ implies firstly that $d$ is a differential, and secondly that the complex 
$\left(\mathcal{C}\left(\mathcal{D} ; \Lambda_{R}\right), d=0\right)$ is identical to the Morse complex associated to $f_{\xi}$. Hence the map

$$
\mathrm{OH}\left(\mathcal{D} ; \Lambda_{R}\right) \rightarrow H M\left(f_{\xi}\right) \otimes \Lambda_{R}
$$

induced by mapping a quantum homology class $[x]_{Q}$ represented by some $x \in$ Crit $f_{\xi}$ to the class $[x]_{M}$ represented by $x$ in Morse homology gives rise to an isomorphism

$$
\mathrm{OH}\left(R ; \Lambda_{R}\right) \cong H\left(R ; \mathbb{Z}_{2}\right) \otimes \Lambda_{R}
$$

We will explain below why this isomorphism is canonical, in the sense that the isomorphisms of type (2) are compatible with the comparison isomorphisms relating different choices of data.

Proof of the Claim. Let $x, y \in$ Crit $f_{\xi}$, let $\lambda \in H_{2}^{D}(X, R) / \sim$ be such that $|x|-|y|+\mu(\lambda)=0$ and consider the involution

$$
\tau_{*}^{\mathcal{P}}: \mathcal{P}(x, y, \lambda) \rightarrow \mathcal{P}(x, y, \lambda), \quad\left(u_{1}, \ldots, u_{k}\right) \mapsto\left(\tau_{*} u_{1}, \ldots, \tau_{*} u_{k}\right)
$$

on the space of pearly trajectories, where on the right $\tau_{*}$ denotes the involution on $\mathcal{M}_{2}$ defined in Section 6. Observe that

$$
\#_{\mathbb{Z}_{2}} \mathcal{P}(x, y, \lambda)=\#_{\mathbb{Z}_{2}} \operatorname{Fix} \tau_{*}^{\mathcal{P}}
$$

Hence $d=0$ will follow if we can show that the number of fixed points of $\tau_{*}^{\mathcal{P}}$ is even. So suppose that $\left(u_{1}, \ldots, u_{k}\right) \in \mathcal{P}(x, y, \lambda)$ is a fixed point of $\tau_{*}^{\mathcal{P}}$, which is equivalent to all $u_{i}$ being fixed points of $\tau_{*}: \mathcal{M}_{2} \rightarrow \mathcal{M}_{2}$. By Lemma 6.1 and the discussion in Section 6.2 we can write

$$
\left(u_{1}, \ldots, u_{k}\right):=\left(\mathfrak{D} v_{1,+}, \ldots, \mathfrak{D} v_{k,+}\right)
$$

with real rational curves $v_{i}: \mathbb{C} P^{1} \rightarrow X$ (recall that $v_{+}$denotes the upper half of $\left.v: \mathbb{C} P^{1} \rightarrow X\right)$. We will argue below that all $v_{i}$ have to be simple. Assuming this for the moment, consider the pearly trajectory

$$
\left(u_{1}^{\prime}, \ldots, u_{k}^{\prime}\right):=\left(\mathfrak{D} v_{1,-}, \ldots, \mathfrak{D} v_{k,-}\right) .
$$


By Proposition 6.2(i), we have $\mathfrak{D} v_{i,+} \neq \mathfrak{D} v_{i,-}$ in $\mathcal{M}_{2}$ and consequently

$$
\left(u_{1}, \ldots, u_{k}\right) \neq\left(u_{1}^{\prime}, \ldots, u_{k}^{\prime}\right)
$$

in $\mathcal{P}(x, y, \lambda)$. This shows that all fixed points of $\tau_{*}^{\mathcal{P}}$ occur in pairs, so that their total number is even.

It remains to justify the simplicity assumption. Consider an element of $\mathcal{P}(x, y, \lambda)$ containing a real disc $u=\mathfrak{D} v_{+}$fixed by $\tau_{*}: \mathcal{M}_{2} \rightarrow \mathcal{M}_{2}$. If $v$ were nonsimple, we could infer by Proposition 6.2(ii) that there exists some real disc $u^{\prime}$ with

$$
\mu\left(u^{\prime}\right)<\mu(u) \quad \text { and } \quad u^{\prime}(D)=u(D) .
$$

Replacing $u$ by $u^{\prime}$ would hence yield a new pearly trajectory representing a homology class $\lambda^{\prime}$ of Maslov number strictly lower than $\mu(\lambda)$. But this is not possible, because under our genericity assumptions $\mathcal{P}\left(x, y, \lambda^{\prime}\right)$ is a manifold of dimension

$$
|X|-|y|+\mu\left(\lambda^{\prime}\right)-1<0
$$

and thus empty. We conclude that $v$ is simple.

It remains to address the question of why the isomorphism $\mathrm{QH}\left(R ; \Lambda_{R}\right) \rightarrow$ $H\left(R ; \mathbb{Z}_{2}\right) \otimes \Lambda_{R}$ to which the family of isomorphisms (2) gives rise is canonical. What we mean by that is that all diagrams of the form

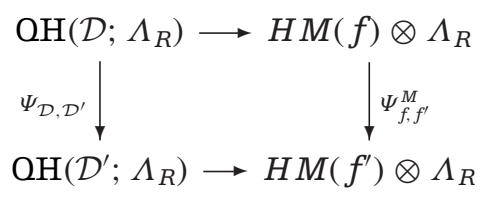

commute, where the horizontal arrows are as in (2) and the vertical arrows are the comparison isomorphisms in quantum respectively Morse homologies. In general, $\Psi_{\mathcal{D}^{\prime}, \mathcal{D}}$ : $\mathrm{OH}\left(\mathcal{D} ; \Lambda_{R}\right) \rightarrow \mathrm{OH}\left(\mathcal{D}^{\prime} ; \Lambda_{R}\right)$ is of the form

$$
\Psi_{\mathcal{D}^{\prime}, \mathcal{D}}=\Psi_{f^{\prime}, f}^{M}+\Psi_{\mathcal{D}^{\prime}, \mathcal{D}}^{Q} t
$$


where $\Psi_{\mathcal{D}^{\prime}, \mathcal{D}}^{Q}$ counts certain configurations containing holomorphic discs (see [6, 7] for details). So the potential source of noncanonicality is that $\Psi_{\mathcal{D}^{\prime}, \mathcal{D}}^{Q}$ might not vanish. However, in our specific case a cancellation argument as in the proof of the claim shows

$$
\Psi_{\mathcal{D}^{\prime}, \mathcal{D}}^{Q}=0
$$

and hence our isomorphism is canonical. This ends the proof of Theorem A(i).

\subsection{Proof of Theorem A(ii)}

As in the proof of the wideness part we work with data triples of the form $\mathcal{D}=\left(f_{\xi}, g_{0}, J_{0}\right)$, and when the product is concerned with a second triple $\mathcal{D}^{\prime}=\left(\tilde{f}_{\xi^{\prime}}, g_{0}, J_{0}\right)$, where $f_{\xi}$, $f_{\xi^{\prime}} \in \mathcal{F}_{0}$.

We already know that $\mathrm{OH}\left(R ; \Lambda_{R}\right)$ and $\mathrm{OH}\left(X ; \Lambda_{X}\right)$ are isomorphic to $H\left(R ; \mathbb{Z}_{2}\right) \otimes$ $\Lambda_{R}$, respectively, $H\left(X ; \mathbb{Z}_{2}\right) \otimes \Lambda_{X}$ and hence isomorphic to each other as modules. Explicitly, a (degree-doubling) isomorphism is induced by

$$
\mathcal{C}(R ; \mathcal{D}) \rightarrow \mathcal{C}(X ; \mathcal{D}), \quad X \mapsto X,
$$

where $f_{\xi}: X \rightarrow \mathbb{R}$ is a generic Morse function of the type described in Section 7, and by the degree-doubling ring isomorphism

$$
\Lambda_{R} \rightarrow \Lambda_{X}
$$

determined by $t \mapsto q\left(\right.$ recall that $\left.|q|=-2 C_{L}=-2 N_{R}=2|t|\right)$. Here we denote by $\mathcal{C}(R ; \mathcal{D})=$ $\left(\mathbb{Z}_{2}\left\langle\right.\right.$ Crit $\left.\left.f_{\xi}\right\rangle \otimes \Lambda_{R}, d=0\right)$ the Lagrangian quantum complex and by $\mathcal{C}(X ; \mathcal{D})$ the complex $\left(\mathbb{Z}_{2}\left\langle\right.\right.$ Crit $\left.f_{\xi}\right\rangle \otimes \Lambda_{X}, d=0$ ) whose homology is $\mathrm{QH}\left(X ; \Lambda_{X}\right)$. It follows from the discussion at the end of the proof of Theorem A(i) that this isomorphism is canonical.

It remains to show that the isomorphism is compatible with the quantum products on both sides. We recall from $[6,7]$ that the quantum product on $\mathrm{OH}\left(R ; \Lambda_{R}\right)$ is by definition induced by the $\Lambda_{\mathbb{Z}_{2}}$-linear homomorphism

$$
\mathcal{C}(R ; \mathcal{D}) \otimes \mathcal{C}\left(R ; \mathcal{D}^{\prime}\right) \rightarrow \mathcal{C}(R ; \mathcal{D}), \quad x \otimes y \mapsto x * y
$$

given by

$$
x * y:=\sum_{z, \lambda}\left(\#_{\mathbb{Z}_{2}} \mathcal{Y}^{D}(x, y, z, \lambda)\right) z t^{\mu(\lambda) / N_{R}},
$$


where $x, y, z$, and $\lambda \in H_{2}^{D}(X, R)$ are such that $|x|+|y|-|z|+\mu(\lambda)-n=0$. Similarly, the quantum product on $\mathrm{OH}\left(X ; \Lambda_{X}\right)$ is defined by $\mathbb{Z}_{2}$-counting elements in the moduli spaces

$$
\mathcal{Y}^{S}(x, y, z ; \lambda)
$$

of spherical pearls described in Section 7, with critical points $x, y, z$, and $\lambda \in H_{2}^{S}(X)$.

Observe that there is a 1-1 correspondence between the sets $H_{2}^{D}(X, R) / \sim$ and $H_{2}^{S}(X) / \sim$ induced by mapping the class $\lambda$ represented by some disc to the class $\lambda^{\sharp}$ represented by its double (recall that $\sim$ identifies classes of the same Maslov respectively Chern numbers). The theorem will hence follow if we can show

$$
\#_{\mathbb{Z}_{2}} \mathcal{Y}^{D}(x, y, z, \lambda)=\#_{\mathbb{Z}_{2}} \mathcal{Y}^{S}\left(x, y, z, \lambda^{\sharp}\right) .
$$

To do so, we begin by decomposing the moduli space $\mathcal{Y}^{D}(x, y, z, \lambda)$ as

$$
\mathcal{Y}^{D}(x, y, z, \lambda)=\mathcal{Y}_{0}^{D}(x, y, z, \lambda) \sqcup \mathcal{Y}_{1}^{D}(x, y, z, \lambda),
$$

where $\mathcal{Y}_{0}^{D}(x, y, z, \lambda)$ is the subset consisting of all those Y-pearls containing some nonconstant noncentral disc, so that $\mathcal{Y}_{1}^{D}(x, y, z, \lambda)$ is the subset of those Y-pearls containing at most a central disc.

Claim 1. $\#_{\mathbb{Z}_{2}} \mathcal{Y}_{0}^{D}(x, y, z, \lambda)=0$.

Proof. Consider the involution

$$
\tau_{*}^{\mathcal{Y}^{S}}: \mathcal{Y}^{D}(x, y, z, \lambda) \rightarrow \mathcal{Y}^{D}(x, y, z, \lambda)
$$

given by reflecting all discs in a product pearl except the central one (note that reflecting the central one would lead to an element in the moduli space $\mathcal{Y}^{S}(y, x, z, \lambda)$ with $x$ and $y$ reversed). Using precisely the same arguments as in the proof of Theorem A(i) one shows that all fixed points of $\tau_{*}^{\mathcal{Y}^{S}}$ occur in pairs, which proves the claim.

Claim 2. $\#_{\mathbb{Z}_{2}} \mathcal{Y}_{1}^{D}(x, y, z, \lambda)=\#_{\mathbb{Z}_{2}} \mathcal{Y}^{S}\left(x, y, z, \lambda^{\sharp}\right)$. 
Proof of Claim 2. There is an obvious map

$$
\mathcal{Y}_{1}^{D}(x, y, z, \lambda) \rightarrow \mathcal{Y}^{S}\left(x, y, z, \lambda^{\sharp}\right)
$$

given by replacing the central disc $u$ of any Y-pearl by its double $u^{\sharp}$.

We argue that this map is injective. Suppose $u_{0} \neq u_{1}$ and $u_{0}^{\#}=u_{1}^{\#}$. Then we must have $u_{1}=\tau \circ u_{0} \circ C$ (the reflection of $u_{0}$ ), and in particular

$$
u_{1}\left(\mathrm{e}^{-2 \pi \mathrm{i} / 3}\right)=u_{0}\left(\mathrm{e}^{2 \pi \mathrm{i} / 3}\right) \quad \text { and } \quad u_{1}\left(\mathrm{e}^{2 \pi \mathrm{i} / 3}\right)=u_{0}\left(\mathrm{e}^{-2 \pi \mathrm{i} / 3}\right)
$$

Since $u_{0}$ appears in a Y-pearl, we may assume $u_{0}\left(\mathrm{e}^{2 \pi \mathrm{i} / 3}\right) \neq u_{0}\left(\mathrm{e}^{-2 \pi \mathrm{i} / 3}\right)$ under our genericity assumptions, so that we obtain $u_{0}\left(\mathrm{e}^{2 \pi \mathrm{i} / 3}\right) \neq u_{1}\left(\mathrm{e}^{2 \pi \mathrm{i} / 3}\right)$ and hence $u_{0}^{\sharp}\left(\mathrm{e}^{2 \pi \mathrm{i} / 3}\right) \neq u_{1}^{\sharp}\left(\mathrm{e}^{2 \pi \mathrm{i} / 3}\right)$, contradicting $u_{0}^{\sharp}=u_{1}^{\sharp}$.

The only Y-pearls which are not in the image of this map are those which are not $\tau$-invariant, in the sense that they contain a sphere which is not a real rational curve or a Morse trajectory which is not entirely contained in $R$. But for these it is easy to see that they occur in pairs and thus do not contribute to the quantum product.

From the two claims we conclude that

$$
\begin{aligned}
\#_{\mathbb{Z}_{2}} \mathcal{Y}^{D}(x, y, z ; \lambda) & =\#_{\mathbb{Z}_{2}} \mathcal{Y}_{0}^{D}(x, y, z ; \lambda)+\#_{\mathbb{Z}_{2}} \mathcal{Y}_{1}^{D}(x, y, z ; \lambda) \\
& =\#_{\mathbb{Z}_{2}} \mathcal{Y}_{1}^{D}(x, y, z ; \lambda) \\
& =\#_{\mathbb{Z}_{2}} \mathcal{Y}^{S}\left(x, y, z ; \lambda^{\sharp}\right),
\end{aligned}
$$

which ends the proof of Theorem A(ii).

\section{Acknowledgements}

The author wishes to thank his advisor, Professor Paul Biran, for suggesting to start this investigation and for plenty of helpful discussions on the subject. He also wishes to thank the anonymous referee for his useful remarks.

\section{Funding}

This work was supported by the Swiss National Science Foundation (grant number 200021_134747). 


\section{References}

[1] Abreu, M. and L. Macarini. "Remarks on Lagrangian intersections in toric manifolds." Transactions of the American Mathematical Society (2011): preprint arXiv:1105.0640 [math.SG].

[2] Alston, G. and L. Amorim. "Floer cohomology of torus fibers and real Lagrangians in Fano toric manifolds." International Mathematical Research Notices (2010): preprint arXiv:1003.3651 [math.SG].

[3] Audin, M. Torus Actions on Symplectic Manifolds. Progress in Mathematics 93. Basel: Birkhäuser, revised edition, 2004.

[4] Audin, M., A. C. da Silva, and E. Lerman. Symplectic Geometry of Integrable Hamiltonian Systems. Advanced Courses in Mathematics. CRM Barcelona. Basel: Birkhäuser, 2003. Lectures delivered at the Euro Summer School held in Barcelona, July 10-15, 2001.

[5] Batyrev, V. V. “Quantum cohomology rings of toric manifolds." Astérisque 218 (1993): 9-34. Journées de Géométrie Algébrique d'Orsay (Orsay, 1992).

[6] Biran, P. and O. Cornea. "Ouantum Structures for Lagrangian Submanifolds." (2007): preprint arXiv:0708.4221 [math.SG].

[7] Biran, P. and O. Cornea. "A Lagrangian Quantum Homology." In New Perspectives and Challenges in Symplectic Field Theory, 1-44. CRM Proceedings \& Lecture Notes 49. Providence, RI: American Mathematical Society, 2009.

[8] Biran, P. and O. Cornea. "Rigidity and uniruling for Lagrangian submanifolds." Geometry and Topology 13, no. 5 (2009): 2881-989.

[9] Bredon, G. E. Introduction to Compact Transformation Groups. Pure and Applied Mathematics 46. New York: Academic Press, 1972.

[10] Cho, C.-H. and Y.-G. Oh. "Floer cohomology and disc instantons of Lagrangian torus fibers in Fano toric manifolds." The Asian Journal of Mathematics 10, no. 4 (2006): 773-814.

[11] Cieliebak, K. and D. Salamon. "Wall crossing for symplectic vortices and quantum cohomology." Mathematische Annalen 335, no. 1 (2006): 133-92.

[12] Delzant, T. "Hamiltoniens périodiques et images convexes de l'application moment." Bulletin de la Société Mathématique de France 116, no. 3 (1988): 315-39.

[13] Duistermaat, J. J. “Convexity and tightness for restrictions of Hamiltonian functions to fixed point sets of an antisymplectic involution." Transactions of the American Mathematical Society 275, no. 1 (1983): 417-29.

[14] Floyd, E. E. "On periodic maps and the Euler characteristics of associated spaces." Transactions of the American Mathematical Society 72 (1952): 138-47.

[15] Frauenfelder, U. "The Arnold-Givental conjecture and moment Floer homology." International Mathematics Research Notices 42 (2004): 2179-269.

[16] Fukaya, K., Y.-G. Oh, H. Ohta, and K. Ono. "Anti-symplectic involution and floer cohomology." (2009): preprint arXiv:0912.2646 [math.SG].

[17] Fukaya, K., Y.-G. Oh, H. Ohta, and K. Ono. Floer theory of Lagrangian submanifolds over $\mathbb{Z}$. http://www.math.kyoto-u.ac.jp/ fukaya/Chap82009ref.pdf. 
[18] Grauert, H. "Analytische Faserungen über holomorph-vollständigen Räumen." Mathematische Annalen 135 (1958): 263-73.

[19] Gunning, R. “Function Theory of Compact Riemann Surfaces." Book draft, 2008. http://www. math.princeton.edu/ gunning/.

[20] Hutchings, M. Lecture notes on Morse homology (with an eye on towards Floer theory and holomorphic curves). http://math.berkeley.edu/ hutching/.

[21] Katz, S. and C.-C. Melissa Liu. "Enumerative geometry of stable maps with Lagrangian boundary conditions and multiple covers of the disc." Advances in Theoretical and Mathematical Physics 5, no. 1 (2001): 1-49.

[22] McDuff, D. and D. Salamon. J-holomorphic Curves and Symplectic Topology. American Mathematical Society Colloquium Publications 52. Providence, RI: American Mathematical Society, 2004.

[23] Oda, T. Convex Bodies and Algebraic Geometry-Toric Varieties and Applications. I. Algebraic Geometry Seminar (Singapore, 1987), 89-94. Singapore: World Scientific Publishing, 1988.

[24] Oh, Y.-G. "Floer cohomology of Lagrangian intersections and pseudo-holomorphic disks. II. $\left(\mathrm{CP}^{n}, \mathrm{RP}^{n}\right) . "$ Communications on Pure and Applied Mathematics 46, no. 7 (1993): 995-1012. 